\title{
ISOSPECTRAL PAIRS OF METRICS ON BALLS, SPHERES, AND OTHER MANIFOLDS WITH DIFFERENT LOCAL GEOMETRIES
}

\author{
Z.I. SZABÓ
}

January 2000

\begin{abstract}
The first isospectral pairs of metrics are constructed on the most simple simply connected domains, namely, on balls and spheres. This long standing problem, concerning the existence of such pairs, has been solved by a new method called "Anticommutator Technique". Among the wide range of such pairs, the most Striking Examples are provided on the spheres $S^{4 k-1}$, where $k \geq 3$. One of these metrics is homogeneous (since it is the metric on the geodesic sphere of a 2-point homogeneous space), while the other is locally inhomogeneous. These examples demonstrate the surprising fact that no information about the isometries is encoded in the spectrum of Laplacian acting on functions. In other words, "The group of isometries, even the local homogeneity property, is lost to the "Non-Audible" in the debate of "Audible versus Non-Audible Geometry"."
\end{abstract}

To the memory of my son Dániel (1976-1999)

Research in Spectral Geometry has been booming in the last two decades. Since Milnor's [M,1964] first example of a pair of 16 dimensional isospectral but non-isometric flat tori, many new examples were constructed. See some of the most important results in [V,1980]; [I,1980]; [GW,1984,1986]; [S,1985]; [Bu,1986]; [BT,1987] [DG,1989]; [GWW,1992], etc. However, all of these previously constructed isospectral pairs consist of locally isometric spaces and they differ from each other just in global shape; global isometries do not exist in these cases but local isometries do.

1991 Mathematics Subject Classification. Primary 58G25; Secondary 53C20, 22E25.

Key words and phrases. Spectral Geometry, isospectral pairs, Laplacian.

Research partially supported by the CUNY grants 9-92720 and 9-91909.

Typeset by $\mathcal{A} \mathcal{M} \mathcal{S}-\mathrm{T}_{\mathrm{E}} \mathrm{X}$ 
The first examples of isospectral metrics with different local geometries were constructed by the author [Sz3,1992] and by C. Gordon [G1,1993]. In these new examples Gordon covered the closed case while the author constructed metrics on the product of a ball by a torus (the boundary of this manifold is the product of a sphere by the same torus) such that the metrics were both Dirichlet and Neumann isospectral. Yet the spaces were locally non-isometric.

To the construction of these examples we used special 2-step nilpotent Lie algebras. In the following we briefly introduce some of those basic notions what are really needed for a clear exposition.

A general metric 2-step nilpotent Lie algebra is described by the system

$$
\left\{\mathbf{n}=\mathbf{v} \oplus \mathbf{z},\langle,\rangle, \mathbf{J}_{Z}\right\},
$$

where $\mathbf{z}=[\mathbf{n}, \mathbf{n}]$ is the center of the Lie algebra, $\langle$,$\rangle is an inner product on \mathbf{n}$ such that $\mathbf{v} \perp \mathbf{z}$ furthermore the one-to-one mapping $\mathbf{J}: \mathbf{z} \rightarrow \operatorname{End}(\mathbf{v})$ is defined by the formula

$$
\left\langle\mathbf{J}_{Z}(X), Y\right\rangle=\langle[X, Y], Z\rangle \quad, \quad \forall X, X^{\prime} \in \mathbf{v} \quad, \quad \forall Z \in \mathbf{z} .
$$

The space $\mathbf{v}$ resp. $\mathbf{z}$ is called also $\mathrm{X}$-space resp. $Z$-space. The linear space of the endomorphisms $\mathbf{J}_{Z}, \forall Z \in \mathbf{z}$ is denoted by $\mathbf{J}_{\mathbf{z}}$. Special 2-step nilpotent Lie algebras are the so called Heisenberg-type algebras, characterized by the property $\mathbf{J}_{Z}^{2}=-|Z|^{2} i d, \forall Z \in \mathbf{z}[\mathrm{K}]$.

Any 2-step nilpotent Lie group $N$ can be extended into a solvable group $S N$ defined on the half space $\mathbf{n} \times \mathbf{R}_{+}$. The corresponding formulas of this extension are described in (3.16) and (3.17). The natural invariant metrics on the solvable extensions of the Heisenberg-type groups are harmonic [DR]. One can find more about harmonic manifolds in [Sz1,2,3].

In case of the special 2-step nilpotent Lie algebra $\mathbf{H}_{3}^{(a, b)}$, used for constructing the above new examples, the $Z$-space is $\mathbf{R}^{3}$ (considered as the space of imaginary quaternions) and the $X$-space is the $4(a+b)$ - dimensional quaternionic space $\mathbf{H}^{a+b}$, where $\mathbf{H}=\mathbf{R}^{4}$ is the space of quaternions and $a, b \in \mathbf{N}$ are natural numbers. The inner product on $\mathbf{n}$ is the natural inner product of the space $\mathbf{n}=\mathbf{R}^{4(a+b)} \times \mathbf{R}^{3}$. The endomorphisms $\mathbf{J}_{Z}, Z \in \mathbf{R}^{3}$ are introduced by using both left and right products as follows:

$$
\mathbf{J}_{Z}^{(a, b)}\left(X_{1}, \ldots, X_{a}, X_{a+1}, \ldots, X_{a+b}\right)=\left(X_{1} Z, \ldots, X_{a} Z, Z X_{a+1}, \ldots, Z X_{a+b}\right) .
$$

The corresponding Lie algebras and Lie groups are denoted by $\mathbf{h}_{3}^{(a, b)}$ and $\mathbf{H}_{3}^{(a, b)}$. The metric defined by the left-invariant extension of the inner product $\langle$,$\rangle of the tangent space$ $\mathbf{h}_{3}^{(a, b)}=T_{0}\left(H_{3}^{(a, b)}\right)$ onto the group is denoted by $g^{(a, b)}$.

The spectral investigations will be performed on four type of domains. These are the Ball $\times$ Torus-type domains, the pure Ball-type domains, and also their Sphere $\times$ Torus- resp. the pure Sphere-type boundaries of these domains will be considered. They are described as follows.

Let $\Gamma_{Z}$ be a full lattice (discrete subgroup) on the Z-space and let $B_{\delta}$ be the euclidean ball of radius $\delta$ around the origin of the X-space. The factor manifold $\Gamma_{Z} \backslash \mathbf{H}_{3}^{(a, b)}$ can be considered as a principal fiber bundle such that the fibers are naturally identified with the torus $T^{3}=\Gamma / \mathbf{R}^{3}$ and the base space is naturally identified with the $\mathrm{X}$-space. When the fibers are considered only over $B_{\delta}$, the bundle is denoted by $\left(B_{\delta}, T^{3}\right)$. The boundary of this manifold is the principal fiber bundle $\left(S_{\delta}, T^{3}\right)$, where the sphere $S_{\delta}=\partial B_{\delta}$ is the base space of this bundle. This construction can be established on any 2-step nilpotent group. 
Besides the above considered Ball $\times$ Torus- and Sphere $\times$ Torus-cases we consider also such compact domains whose boundaries are described by equations of the form $f(|X|,|Z|)=0$. In these cases the domains are diffeomorphic to euclidean balls and the boundaries are diffeomorphic to euclidean spheres. We call these cases Ball-cases and Sphere-cases.

The corresponding domains and their boundaries on the solvable extensions are introduced by a natural modification.

Then we have

Theorem [Sz3]. The metrics $g^{(a, b)}$ with the same $(a+b)$ but with different $a b$ are locally non-isometric, yet they are both Dirichlet and Neumann isospectral on the manifolds $\left(B_{\delta}, T^{3}\right)$.

Beside proving the isospectral property by constructing an intertwining operator, also an explicit computation of the spectrum is provided in the paper.

Gordon and Wilson generalized this construction [GW3] to obtain continuous families of isospectral metrics such that the family members have different local geometries (namely the scalar curvature is different on these spaces). Their Main Theorem states

Theorem [GW3]. Let $\left\{\mathbf{n}=\mathbf{v} \oplus \mathbf{z},\langle\rangle,, \mathbf{J}_{Z}\right\}$ and $\left\{\mathbf{n}^{\prime}=\mathbf{v} \oplus \mathbf{z},\langle\rangle,, \mathbf{J}^{\prime}{ }_{Z}\right\}$ be Lie algebras defined over the same space $\mathbf{v} \oplus \mathbf{z}$ such that $\mathbf{J}_{Z}$ and $\mathbf{J}_{Z}^{\prime}$ are isospectral (conjugate), $\forall Z \in \mathbf{z}$. Then the left-invariant metrics $g$ and $g^{\prime}$ are both Dirichlet and Neumann isospectral on any of the Ball $\times$ Torus-type manifolds described above.

Notice that the endomorphisms introduced in (0.3) are spectrally equivalent in the sense of the above theorem on spaces with the same $a+b$. It should be mentioned that the existence of non-trivial isospectral deformations is proven in [GW3] only on spaces where this torus is 2-dimensional. It is still an open question if this construction provides non-trivial continuous isospectral deformations on spaces having higher dimensional Z-spaces. This points out one of the differences between the constructions of [GW3] and [Sz3]. It should be emphasized too that this generalization strictly concerns the Ball $\times$ Torus-type manifolds and it is not valid, for instance, on pure Ball-type domains.

Further developed this field by Gordon, Gornet, Schueth, Webb and Wilson [GGSWW] by noticing that the metrics $g_{t}$ restricted to the boundary $S_{\delta} \times T^{2}$ of the spaces constructed in [GW3] are isospectral with different local geometries. The new feature of these examples is that these spaces are closed and locally inhomogeneous.

Independently also this author noticed that the metrics $g^{(a, b)}$ restricted 
to the boundary $S_{\delta} \times T^{3}$ of his examples are isospectral with different local geometries. (This observation is included in a revised version of the article [Sz3].) The new feature of these examples is that one of these spaces (belonging to $g^{(0, a+b)}$ ) is homogeneous while the other spaces (belonging to $g^{(a, b)}$, where $a b \neq 0$ ) are locally inhomogeneous. This is the first demonstration of the fact that one can not hear the local homogeneity property of closed Riemannian manifolds.

Recently D. Schueth [Sch] constructed continuous families of isospectral Riemannian metrics on simply connected manifolds such that the different members of a family have different scalar curvature. In her construction she enlarges the torus $T^{2}$ of the spaces considered in [GGSWW] into a compact simply connected Lie group $G$ such that $T^{2}$ is a maximal torus of $G$. Then she obtains her examples by an extension of the metrics $g_{t}$ of $S_{\delta} \times T^{2}$ onto the enlarged manifold $S_{\delta} \times G$. In special cases she gets examples on the product of spheres. The space with the lowest dimension is constructed on $S^{4} \times S^{3} \times S^{3}$. These are the first examples for isospectral non-isometric metrics constructed on closed simply connected manifolds.

Most recently Gordon and this author [GS] constructed continuous families of negatively curved manifolds with boundaries such that the metrics of the distinct family members are isospectral, yet having different local geometries. This result contrasts the Rigidity Theorem of Crocke and Sharafutdinov $[\mathrm{CS}]$, asserting that such continuous deformations do not exist on closed manifolds.

It should be mentioned that all the above constructions involve only the spectrum of the Laplacian acting on functions. The manifolds used for these constructions are exclusively the manifolds described in the Ball $\times$ Toruscases or in the Sphere $\times$ Torus-cases. No examples of non-trivial isospectral pairs are constructed on the most simple simply connected manifolds, namely, on balls or on spheres so far.

Examples concerning the spectra of forms or spectrum of the Dirac operator are also completely missing from the literature. The most far reaching statement from this point of view is that the metrics constructed by Schueth are not isospectral on 1-forms [Sch].

We extend our investigations in these directions. The main goal of this paper is to find the first non-trivial isospectral pairs of spaces constructed on the most simple domains, namely, on balls and spheres. However, we scrutinize also the question about the importance of using spectrally equivalent endomorphism spaces for constructing isospectral metrics. It turns out that this property is important in case of Ball $\times$ Torus-type domains 
and on their boundaries but it is not important, at all, on the Ball-type domains and on their Sphere-type boundaries. A brief outline of these considerations is as follows.

First discrete-type, so called $\sigma$-deformations are introduced on 2-step nilpotent Lie algebras. These deformations are generalizations of the special deformations used for constructing the non-isomorphic Lie algebras $\mathbf{h}_{3}^{(a, b)}$ in (0.3). Also these generalized deformations provide spectrally equivalent endomorphism metrics. The more precise details are as follows.

Consider a Lie algebra $\left\{\mathbf{n}=\mathbf{v} \oplus \mathbf{z},\langle\rangle,, \mathbf{J}_{Z}\right\}$ and let $\sigma$ be an involutive orthogonal transformation on $\mathbf{v}$ commuting with the endomorphisms $\mathbf{J}_{Z}, \forall Z \in \mathbf{z}$. Then the $\sigma$-deformation of this Lie algebra is defined by $\left\{\mathbf{n}_{\sigma}=\mathbf{v} \oplus \mathbf{z},\langle\rangle,, \mathbf{J}_{Z}^{\sigma}\right\}$, where only the endomorphisms $\mathbf{J}_{Z}^{\sigma}$ are different in the new system and they are defined by $\mathbf{J}_{Z}^{\sigma}=\sigma \mathbf{J}_{Z}, \forall Z \in \mathbf{z}$. The corresponding left invariant metrics on the $\sigma$-deformed groups are denoted by $g^{\sigma}$.

More general deformations are the so called Partial $\sigma$-deformations (they are called also $P \sigma$-deformations). In this case we perform $\sigma$-deformations only on the elements of a subspace $\mathbf{J}_{\mathbf{s}} \subset \mathbf{J}_{\mathbf{z}}$, while keeping the orthogonal complement $\mathbf{J}_{\mathbf{s}^{\perp}}$ unchanged.

Though the Partial $\sigma$-deformations form a much wider class than the $\sigma$-deformations, in very particular cases a $\sigma$-deformation can be equivalent to a $P \sigma$-deformation. This is the case when the endomorphism space contains at least a 1-dimensional subspace, $\mathbf{J}_{\mathbf{A}}$, of the so called anticommutators. A non-degenerated endomorphism $A \in \mathbf{J}_{\mathbf{z}}$ is an anticommutator iff $A \circ B=-B \circ A, \forall B \in \mathbf{J}_{A^{\perp}}$. A Partial $\sigma$-deformation established by the deformation $A^{\sigma}$ of an anticommutator $A$, is called $\sigma_{A}$-deformation. In Reduction Theorem 4.1 we prove that a $\sigma$-deformation is always equivalent to the $\sigma_{A}$-deformation, if the endomorphism space contains an anticommutator $A$.

The general $\sigma$-deformations provide isospectral endomorphisms in the sense of Theorem [GW3], therefore they provide a wide range of new examples for non-trivial isospectral pairs on the Ball $\times$ Torus-type domains. However, the general sigma-deformations do not provide isospectral metrics on the pure Ball-type domains and on their Sphere-type boundaries. Our main goal in this paper is to find "such/those" sigma-deformations which lead to isospectral metrics also on these simple domains. It turns out that the particular $\sigma_{A}$-deformations are "such" deformations. More precisely, we have 
Main Theorem 4.3. Let $\mathbf{J}_{\mathbf{z}}=\mathbf{A} \oplus \mathbf{A}^{\perp}$ be an Endomorphism Space with the anticommutator $A$ and let $\sigma$ be an involutive orthogonal transformation on the $X$-space commuting with all of the endomorphisms from $\mathbf{J}_{\mathbf{z}}$. Then the metrics $g$ and $g^{\sigma}$, determined by the Endomorphism Spaces $\mathbf{J}_{\mathbf{z}}$ and $\mathbf{J}_{\mathbf{z}}^{\sigma} \simeq \mathbf{A}^{\sigma} \oplus \mathbf{A}^{\perp}$, are both Dirichlet and Neumann isospectral on the Balltype domains of the considered manifolds.

This Isospectrality Theorem holds also on the Sphere-type boundaries of these domains and both versions of this Theorem can be established also on the solvable extensions of 2-step nilpotent groups.

The Theorem is proven by constructing intertwining operators. It says that, on balls and spheres, isospectral metrics can be constructed by appropriate deformations of an anticommutator in an Endomorphism Space. In this paper we consider mostly the $\sigma_{A}$-deformations of an anticommutator. However, the proofs given in this case make it apparent that they are "working" in a much more general situation, namely, when the anticommutator is deformed only isospectrally. The full exploration of all options for such deformations will be completed in the continuation [Sz4] of this paper. There we discover also a cornucopia of the so called $S p e C h_{A}$-deformations which provide spectrally inequivalent Endomorphism Spaces and therefore a completely new type of examples. These $S p e C h_{A}$-deformations show that our method is drastically forking from all of the methods applied on this field so far. This completely new method is called constructions of isospectral metrics by means of isospectral deformations of an anticommutator in an Endomorphism Space. Or, we simply call it Anticommutator Technique. This new method inherits no "tricks" from the previous investigations performed on this field, since all the tools applied on the Ball $\times$ Torus-type manifolds so far break down on the Ball-type domains and on their Spheretype boundaries.

We provide also the corresponding Non-Isometry Proofs in this paper. Thus a wide range of isospectral pairs of metrics are constructed also on balls and spheres such that the metrics in a pair define different local geometries. By any means, these are the very first examples of isospectral pairs of metrics constructed on these most simple simply connected domains.

The most Striking Examples are constructed on the geodesic spheres of the Harmonic Spaces $S \mathbf{H}_{3}^{(a, b)}$ ). Since the geodesic spheres are homogeneous on the 2-point homogeneous space $S \mathbf{H}_{3}^{(a+b, 0)}$ and the geodesic spheres are inhomogeneous on the other considered harmonic spaces, these exam- 
ples provide isospectral pairs of metrics constructed on a sphere such that one of them is a homogeneous and the other is a locally inhomogeneous metric. These examples demonstrate the fact that the group of isometries is lost to the "Non-Audible" in the debate of "Audible versus Non-Audible Geometry".

The question about the isospectrality of the geodesic balls and spheres on the above harmonic spaces was asked in [Sz2]. The investigations on this field took several unexpected turns, till the Isospectrality Theorem has been established in the general form stated in this paper.

Finally, we would like to mention two related Statements. Each of them is suggestive concerning the possible form of the Isospectrality Theorem considered on forms.

Propositions 5.3-5.4. Two $\sigma$-equivalent metrics have identical Ricci curvature at the origin. However, they have only isotonal Riemannian curvature.

(Two operators are said to be isotonal if the elements of the spectra are the same, but the multiplicities may be different. (Of course, 0-multiplicity is not allowed in this counting.) We use this notion with respect to the Laplacian as well as to the curvature operator $R_{a b}{ }^{c d}$ acting as a symmetric endomorphism on the space of 2 -forms.)

This statement contrasts the following statement:

Proposition 5.1-5.2. Two $\sigma$-equivalent metrics are Laplace-isospectral on the invariant 1- and 2-forms.

(Formula (1.18) shows the involvement of the curvature operator into the Laplacian acting on forms, showing also the contrasting nature of the above two statements.)

This is the opening paper of related articles. Since we did not want to cram to much technical details in one paper, we divided these technicalities between this paper and article [Sz4]. Yet, we tried to make them as independent as it was possible. In this paper the proofs are complete on manifolds with boundaries (i.e. on Ball $\times$ Torus-type and on Ball-type manifolds) and some of the technical details, what are needed on the boundary manifolds, are discussed in [Sz4]. In some extend this remark concerns also the Striking Examples, where the Non-Isometry Proof is based on an explicit computation of the eigenvalues of the Ricci operator. These computation is incorporated into [Sz4]. All the other details are self-contained.

I would like to express my gratitude to Carolyn Gordon for the helpful 
discussions resulting in a clearer exposition and to Attila Máté for helping me in the preparation of this article.

\section{1. $\S$ Two-step nilpotent Lie algebras}

In the Introduction we reviewed some of the basic materials about 2-step nilpotent Lie algebras. They are described by a system

$$
\left\{\mathbf{n}=\mathbf{v} \oplus \mathbf{z},\langle,\rangle, \mathbf{J}_{Z}\right\},
$$

as it is explained in (0.1) and (0.2). A great deal about these Lie algebras can be found in $[\mathrm{E}]$. Such a Lie algebra is well defined by the endomorphisms $\mathbf{J}_{Z}$ and by the inner product $\langle$,$\rangle defined on \mathbf{n}$. The linear space of these endomorphisms is denoted by $\mathbf{J}_{\mathbf{z}}$ and for an $\mathrm{X}$-vector $X \in \mathbf{v}, \mathbf{J}_{\mathbf{z}}(X)$ denotes the linear subspace of $\mathbf{v}$ spanned by the vectors of the form $\mathbf{J}_{Z}(X), \forall Z \in \mathbf{z}$.

The exponential map identifies the Lie group $N$ with the space $\mathbf{v} \oplus \mathbf{z}$ and the group product on $N$ is described by the formula

$$
(X, Z) \cdot\left(X^{\prime}, Z^{\prime}\right)=\left(X+X^{\prime}, Z+Z^{\prime}+1 / 2\left[X, X^{\prime}\right]\right) .
$$

Consider the orthonormal bases $\left\{E_{1} ; \ldots ; E_{k}\right\}$ and $\left\{e_{1} ; \ldots ; e_{l}\right\}$ on the Xand on the Z-space respectively. The corresponding coordinate systems defined by these bases are denoted by $\left\{x^{1} ; \ldots ; x^{k}\right\}$ and $\left\{z^{1} ; \ldots ; z^{l}\right\}$. We extend the vectors $E_{i} ; e_{\alpha}$ into the left-invariant vector fields

$$
\begin{gathered}
\mathbf{X}_{i}=\partial_{i}+\frac{1}{2} \sum_{\alpha=1}^{l}\left\langle\left[X, E_{i}\right], e_{\alpha}\right\rangle \partial_{\alpha}= \\
=\partial_{i}+\frac{1}{2} \sum_{\alpha=1}^{l}\left\langle\mathbf{J}_{\alpha}(X), E_{i}\right\rangle \partial_{\alpha} \quad ; \quad \mathbf{Z}_{\alpha}=\partial_{\alpha},
\end{gathered}
$$

where $\partial_{i}=\partial / \partial x^{i}, \partial_{\alpha}=\partial / \partial z^{\alpha}$ and $\mathbf{J}_{\alpha}=\mathbf{J}_{e_{\alpha}}$.

By using these vector fields, we establish some explicit formulas on 2-step nilpotent Lie groups.

The covariant derivative can be computed by the well known formula

$$
\left\langle\nabla_{P} Q, R\right\rangle=\frac{1}{2}\{\langle P,[R, Q]\rangle+\langle Q,[R, P]\rangle+\langle[P, Q], R\rangle\},
$$

where $P, Q, R$ are considered as elements of the Lie algebra $\mathbf{n}$ (or, they can be considered as invariant vector fields on $N$ ). By this formula we get

$$
\nabla_{X} X^{*}=\frac{1}{2}\left[X, X^{*}\right] ; \nabla_{X} Z=\nabla_{Z} X=-\frac{1}{2} \mathbf{J}_{Z}(X) ; \nabla_{Z} Z^{*}=0 .
$$


The Laplacian $\Delta$ acting on functions can be explicitly computed by using the formula

$$
\Delta=\sum_{i=1}^{k}\left(\mathbf{X}_{i}^{2}-\nabla_{\mathbf{X}_{i}} \mathbf{X}_{i}\right)+\sum_{\alpha=1}^{l}\left(\mathbf{Z}_{\alpha}^{2}-\nabla_{\mathbf{z}_{\alpha}} \mathbf{Z}_{\alpha}\right) .
$$

Then one obtains the following explicit formula

$$
\Delta=\Delta_{X}+\Delta_{Z}+\frac{1}{4} \sum_{\alpha, \beta=1}^{l}\left\langle\mathbf{J}_{\alpha}(X), \mathbf{J}_{\beta}(X)\right\rangle \partial_{\alpha \beta}^{2}+\sum_{\alpha=1}^{l} \partial_{\alpha} D_{\alpha} \bullet
$$

where $D_{\alpha} \bullet$ means differentiation (directional derivative) with respect to the vector field

$$
D_{\alpha}: X \rightarrow \mathbf{J}_{\alpha}(X)
$$

tangent to the X-space, furthermore $\partial_{\alpha \beta}=\partial^{2} / \partial z^{\alpha} \partial z^{\beta}$.

We compute also some other basic objects explicitly. The Riemannian curvature tensor on 2-step nilpotent groups are explicitly computed in [E]. By those formulas we have:

$$
\begin{gathered}
R(X, Y) X^{*}=\frac{1}{2} \mathbf{J}_{[X, Y]}\left(X^{*}\right)-\frac{1}{4} \mathbf{J}_{\left[Y, X^{*}\right]}(X)+\frac{1}{4} \mathbf{J}_{\left[X, X^{*}\right]}(Y) ; \\
R(X, Y) Z=-\frac{1}{4}\left[X, \mathbf{J}_{Z}(Y)\right]+\frac{1}{4}\left[Y, \mathbf{J}_{Z}(X)\right] ; \quad R\left(Z_{1}, Z_{2}\right) Z_{3}=0 ; \\
R(X, Z) Y=-\frac{1}{4}\left[X, \mathbf{J}_{Z}(Y)\right] ; \quad R(X, Z) Z^{*}=-\frac{1}{4} \mathbf{J}_{Z} \mathbf{J}_{Z^{*}}(X) ; \\
R\left(Z, Z^{*}\right) X=-\frac{1}{4} \mathbf{J}_{Z^{*}} \mathbf{J}_{Z}(X)+\frac{1}{4} \mathbf{J}_{Z} \mathbf{J}_{Z^{*}}(X)
\end{gathered}
$$

where $X ; X^{*} ; Y \in \mathbf{v}$ and $Z ; Z^{*} ; Z_{1} ; Z_{2} ; Z_{3} \in \mathbf{z}$ are elements of the Lie algebra.

By introducing $H\left(X, X^{*}, Z, Z^{*}\right):=\left\langle\mathbf{J}_{Z}(X), \mathbf{J}_{Z^{*}}\left(X^{*}\right)\right\rangle$, for the Ricci curvature we have

$$
\begin{gathered}
\operatorname{Ricc}\left(X, X^{*}\right)=-\frac{1}{2} \sum_{\alpha=1}^{l} H\left(X, X^{*}, e_{\alpha}, e_{\alpha}\right) \quad ; \quad \operatorname{Ricc}(X, Z)=0 ; \\
\operatorname{Ricc}\left(Z, Z^{*}\right)=\frac{1}{4} \sum_{i=1}^{k} H\left(E_{i}, E_{i}, Z, Z^{*}\right) .
\end{gathered}
$$


By (1.2), the volume form $\varepsilon$, the metric tensor

$$
g_{i j}=g\left(\partial_{i}, \partial_{j}\right) \quad, \quad g_{i \alpha}=g\left(\partial_{i}, \partial_{\alpha}\right) \quad, \quad g_{\alpha \beta}=g\left(\partial_{\alpha}, \partial_{\beta}\right)
$$

and the inverse metric tensor $g^{i j}, g^{i \alpha}, g^{\alpha \beta}$ have the following explicit forms:

$$
\begin{gathered}
g_{i j}=\delta_{i j}+\frac{1}{4}\left\langle\left[X, \partial_{i}\right],\left[X, \partial_{j}\right]\right\rangle=\delta_{i j}+\frac{1}{4} \sum_{\alpha=1}^{l}\left\langle\mathbf{J}_{\alpha}(X), \partial_{i}\right\rangle\left\langle\mathbf{J}_{\alpha}(X), \partial_{j}\right\rangle ; \\
g_{i \alpha}=-\frac{1}{2}\left\langle\mathbf{J}_{\alpha}(X), \partial_{i}\right\rangle \quad ; \quad g_{\alpha \beta}=\delta_{\alpha \beta},
\end{gathered}
$$

$$
\begin{gathered}
g^{i j}=\delta_{i j} \quad ; \quad g^{i \alpha}=\frac{1}{2}\left\langle\partial_{i}, \mathbf{J}_{\alpha}(X)\right\rangle ; \\
g^{\alpha \beta}=\delta_{\alpha \beta}+\frac{1}{4}\left\langle\mathbf{J}_{\alpha}(X), \mathbf{J}_{\beta}(X)\right\rangle .
\end{gathered}
$$

Finally we describe the Dirac- and Laplace-operators acting on forms.

First we introduce some minor changes in the notations. So far, we used the coordinates $x^{i}$ and $z^{\alpha}$ on the Lie algebra. Next we keep the coordinates $x^{i}$, while we change $z^{\alpha}$ to $x^{k+\alpha}$. These new coordinates are denoted by $x^{A}$, where $A=1, \ldots, k+l$. In the following we use also the Einstein summation convention.

Consider a p-form

$$
\omega=\omega_{\left(A_{1} \ldots A_{p}\right)} d x^{A_{1}} \wedge \cdots \wedge d x^{A_{p}}
$$

where $\left(A_{1} \ldots A_{2}\right)$ implies $A_{1}<\cdots<A_{p}$. The $(n-p)$ )form $* \omega$ (where $n=k+l)$ is defined as usual by

$$
* \omega=\omega^{*}{ }_{\left(B_{1} \ldots B_{n-p}\right)} d x^{B_{1}} \wedge \cdots \wedge d x^{B_{n-p}},
$$

where

$$
\omega^{*} B_{1} \ldots B_{n-p}=\omega^{\left(C_{1} \ldots C_{p}\right)} \varepsilon_{\left(C_{1} \ldots C_{p}\right) B_{1} \ldots B_{n-p}} .
$$


Then we have:

$$
\begin{gathered}
(\delta \omega)_{A_{1} \ldots A_{p-1}}=(-1)^{n p+n+1}(* d * \omega)_{A_{1} \ldots A_{p-1}}= \\
-g^{A B} \delta_{A A_{1} \ldots A_{p-1}}^{\left(B_{1} \ldots B_{p}\right)} \nabla_{B} \omega_{\left(B_{1} \ldots B_{p}\right)},
\end{gathered}
$$

where $\delta_{A_{1} \ldots A_{p}}^{B_{1} \ldots B_{p}}=\operatorname{det}\left(\delta_{A_{i}}^{B_{j}}\right)$ is the multivariable Kronecker symbol.

The Non-Relativistic Dirac- and the Laplace-operator (acting on forms) are defined by

$$
d-\delta \quad \text { and } \quad \Delta=(d-\delta)^{2}=-(d \delta+\delta d)
$$

respectively. Then, for the Laplacian we have the following well known formula:

$$
\begin{gathered}
(\Delta \omega)_{A_{1} \ldots A_{p}}=g^{A B} \nabla_{A} \nabla_{B} \omega_{A_{1} \ldots A_{p}}-\sum_{s=1}^{p} \omega_{A_{1} \ldots A_{s-1} B A_{s+1} \ldots A_{p}} \operatorname{Ricc}_{A_{s}}^{B} \\
-\frac{1}{2} \sum_{s=1}^{p} \sum_{r=1}^{p} \omega_{A_{1} \ldots A_{r-1} B A_{r+1} \ldots A_{s-1} A A_{s+1} \ldots A_{p} R^{A B}{ }_{A_{r} A_{s}} .}
\end{gathered}
$$

We conclude this paragraph by describing the isometries on 2-step nilpotent metric Lie groups.

Proposition $1.1([\mathrm{~K}][\mathrm{E}][\mathrm{W}])$. The 2-step nilpotent metric Lie groups $(N, g)$ and $\left(N^{\prime}, g^{\prime}\right)$ are isometric iff there exist orthogonal transformations $A: \mathbf{v} \rightarrow \mathbf{v}^{\prime}$ and $C: \mathbf{z} \rightarrow \mathbf{z}^{\prime}$ such that

$$
A \mathbf{J}_{Z} A^{-1}=\mathbf{J}_{C(Z)}^{\prime}
$$

holds for any $Z \in \mathbf{z}$.

(First Kaplan established this Statement on H-type groups. Then Wilson generalized it to nilpotent Lie groups. Eberlein noticed, that Kaplan's proof can be applied to 2-step nilpotent case with no altering. See this Statement also in [GW3] as Proposition 1.4.)

As an example we mention, that the metric groups $\mathbf{H}_{3}^{(a, b)}$ (introduced in (0.3)) are isometric to the groups $\mathbf{H}_{3}^{\prime(a, b)}$, where $\mathbf{J}_{Z}^{\prime}$ is defined by

$$
\begin{gathered}
\mathbf{J}^{\prime}{ }_{Z}\left(X_{1}, \ldots, X_{a}, X_{a+1}, \ldots X_{a+b}\right) \\
=\left(X_{1} Z, \ldots, X_{a} Z,-X_{a+1} Z, \ldots,-X_{a+b} Z\right) .
\end{gathered}
$$

In this case $C=i d$ and

$$
A\left(X_{1}, \ldots, X_{a+b}\right)=\left(X_{1}, \ldots, X_{a}, \bar{X}_{a+1}, \ldots, \bar{X}_{a+b}\right),
$$

where $\bar{X}$ means conjugation, establish an isometry between the two spaces. 


\section{2. $\S$ The general $\sigma$ - and $\sigma^{(a+b)}$-deformations}

Let $\left\{\mathbf{n}=\mathbf{v} \oplus \mathbf{z},\langle\rangle,, \mathbf{J}_{Z}\right\}$ be a 2-step nilpotent Lie algebra and let $\sigma$ be an involutive orthogonal transformation on the X-space, commuting with all of the endomorphisms $\mathbf{J}_{Z} \in \mathbf{J}_{\mathbf{z}}$. Then the $\sigma$-deformation of this Lie algebra is defined by the system $\left\{\mathbf{n}_{\sigma}=\mathbf{v} \oplus \mathbf{z},\langle\rangle,, \mathbf{J}_{Z}^{\sigma}\right\}$, where

$$
\mathbf{J}_{Z}^{\sigma}=\sigma \mathbf{J}_{Z}
$$

We call $\mathbf{n}$ and $\mathbf{n}_{\sigma}$ also $\sigma$-equivalent Lie algebras. Notice that only the endomorphisms $\mathbf{J}_{Z}$ (i.e. the Lie brackets) are different in the new system, while the metric structure remains the old one.

All the possible $\sigma$-deformations of $\mathbf{n}$ can be easily described as follows.

Let $\mathbf{v}=\mathbf{v}_{-} \oplus \mathbf{v}_{+}$be an orthogonal direct sum such that both $\mathbf{v}_{-}$and $\mathbf{v}_{+}$are invariant with respect to the action of endomorphisms $\mathbf{J}_{Z} \in \mathbf{J}_{\mathbf{z}}$. The corresponding decomposition of a vector $\mathrm{X}$ is described in the form $X=X_{-}+X_{+}$. Then $\sigma(X)=-X_{-}+X_{+}$is an appropriate transformation for the $\sigma$-deformation of $\mathbf{n}$. If all the invariant subspaces in the irreducible decomposition of the $\mathrm{X}$-space by the action of the Endomorphism Space have greater dimension then 2, then the above construction describes all the $\sigma$-deformations on the Endomorphism Space.

The $\sigma^{(a+b)}$-deformations are special $\sigma$-deformations, which (cf. (0.3) and (1.20)) can be considered as a more straightforward generalization of the concept developed for introducing the Lie algebras $\mathbf{h}_{3}^{(a, b)}$.

Notice that in this special case the left products $L_{Z}: \mathbf{H} \rightarrow \mathbf{H}$ (as well as the right products $R_{Z}: \mathbf{H} \rightarrow \mathbf{H}$ ) by imaginary quaternions $Z$ define an irreducible representation of the Lie algebra $\mathbf{z}=\mathbf{R}^{3}=s o(3)$ on the 4-dimensional space $\mathbf{H}=\mathbf{R}^{4}$.

For generalizing this concept, consider an $l$-dimensional linear subspace $\mathbf{J}_{\mathbf{z}} \subseteq s o(n)$ of orthogonal endomorphisms. Such a subspace can be defined by an appropriate linear map $\mathbf{J}: \mathbf{z}=\mathbf{R}^{l} \rightarrow \operatorname{End}\left(\mathbf{R}^{n}\right)$. The space $\mathbf{J}_{\mathbf{z}}$ may or may not be closed with respect to the commutator bracket of endomorphisms. In the special case of $h_{3}^{(a, b)}$ it corresponds to the space $L_{\mathbf{R}^{3}} \subset s o(4)$ (or to the space $R_{\mathbf{R}^{3}} \subset s o(4)$ ), i. e. $\mathbf{J}=L$ or $\mathbf{J}=R$, where $L$ and $R$ are described above.

For a pair $(a, b)$ of natural numbers, the space $\mathbf{v}$ is introduced by the $(a+b)$-times Cartesian product $\mathbf{v}=\mathbf{R}^{n} \times \cdots \times \mathbf{R}^{n}$. The endomorphisms 
$\mathbf{J}^{(a, b)}: \mathbf{z}=\mathbf{R}^{l} \rightarrow \operatorname{End}(\mathbf{v})$ are defined by

$$
\begin{gathered}
\mathbf{J}_{Z}^{(a, b)}\left(X_{1}, \ldots, X_{a+b}\right) \\
=\left(\mathbf{J}_{Z}\left(X_{1}\right), \ldots, \mathbf{J}_{Z}\left(X_{a}\right),-\mathbf{J}_{Z}\left(X_{a+1}\right), \ldots,-\mathbf{J}_{Z}\left(X_{a+b}\right)\right) .
\end{gathered}
$$

The corresponding Lie algebra defined by these endomorphisms is denoted by $\mathbf{n}_{J}^{(a, b)}$.

If the space $J_{\mathbf{z}}$ is a Lie algebra, then so is the space $\mathbf{J}_{\mathbf{z}}^{(a+b, 0)}$ and these two Lie algebras are isomorphic. However, the space $\mathbf{J}_{\mathbf{z}}^{(a, b)}$ with $a b \neq 0$ can not form a Lie algebra if $J_{\mathbf{z}}$ is a non-Abelian Lie algebra.

In fact, the involution

$$
\sigma\left(X_{1}, \ldots, X_{a+b}\right)=\left(X_{1}, \ldots, X_{a},-X_{a+1}, \ldots,-X_{a+b}\right)
$$

(establishing the deformation $\sigma \mathbf{J}_{\mathbf{z}}^{(a, b)}=\mathbf{J}_{\mathbf{z}}^{(a+b, 0)}$ ) commutes with the elements of both Lie algebras, therefore we have:

$$
\begin{gathered}
{\left[\mathbf{J}_{\mathbf{z}}^{(a, b)}, \mathbf{J}_{\mathbf{z}}^{(a, b)}\right]=\left[\mathbf{J}_{\mathbf{z}}^{(a+b, 0)}, \mathbf{J}_{\mathbf{z}}^{(a+b, 0)}\right] \subseteq \mathbf{J}_{\mathbf{z}}^{(a+b, 0)},} \\
{\left[\mathbf{J}_{\mathbf{z}}^{(a, b)}, \mathbf{J}_{\mathbf{z}}^{(a+b, 0)}\right] \subseteq \mathbf{J}_{\mathbf{z}}^{(a, b)}}
\end{gathered}
$$

Since $\mathbf{J}_{\mathbf{z}}^{(a, b)} \cap \mathbf{J}_{\mathbf{z}}^{(a+b, 0)}=0$, the space $\mathbf{J}_{\mathbf{z}}^{(a, b)}$ can not form a Lie algebra.

In this latter case the space $\mathbf{s}^{(a, b)}=\mathbf{J}_{\mathbf{z}}^{(a, b)} \oplus \mathbf{J}_{\mathbf{z}}^{(a+b, 0)}$ forms a Lie algebra isomorphic to $\mathbf{J}_{\mathbf{z}} \oplus \mathbf{J}_{\mathbf{z}}$. We will show that $\mathbf{s}^{(a, b)}$ and $\mathbf{s}^{\left(a^{\prime}, b^{\prime}\right)}$ with $a+b=a^{\prime}+b^{\prime}$ and $a b \neq a^{\prime} b^{\prime}$ are two inequivalent (non-conjugate) representations of the Lie algebra $\mathbf{J}_{\mathbf{z}} \oplus \mathbf{J}_{\mathbf{z}}$. This is the heart of the following Non-Isometry Proof.

Theorem 2.1. (A) The Lie algebras $\mathbf{n}_{J}^{(a, b)}$ with the same $(a+b)$ and $J$ are $\sigma$-equivalent. Furthermore, the metric Lie group $N_{J}^{(a, b)}$ is isometric to $N_{J}^{(b, a)}$.

(B) If $\mathbf{J}_{\mathbf{z}}$ is a non-Abelian Lie algebra (or more generally, it contains a non-Abelian Lie subalgebra), then $N_{J}^{(a, b)}$ is locally non-isometric to $N_{J}^{\left(a^{\prime}, b^{\prime}\right)}$ unless $(a, b)=\left(a^{\prime}, b^{\prime}\right)$ up to an order.

Proof. (A)The above spaces are clearly $\sigma$-equivalent. Furthermore (cf. Proposition 1.1), $A=i d$ and $C=-i d$ establish an isometry between $N_{J}^{(a, b)}$ and $N_{J}^{(b, a)}$.

(B) First we suppose that $\mathbf{J}_{\mathbf{z}}$ is a Lie algebra. 
If $a b \neq 0$, the spaces $\mathbf{J}_{\mathbf{z}}^{(a, b)}$ and $\mathbf{J}_{\mathbf{z}}^{(a+b, 0)}$ can not be conjugate (see (2.4)). Therefore the metric Lie groups $N_{J}^{(a, b)}$ and $N_{J}^{(a+b, 0)}$ are non-isometric. In fact, if they were isometric, the corresponding spaces of endomorphisms would be conjugate, by Proposition 1.1.

To complete the proof in the general case, consider the derived algebra $\mathbf{J}_{\mathbf{z}^{\prime}}=\left[\mathbf{J}_{\mathbf{z}}, \mathbf{J}_{\mathbf{z}}\right]$ along with the corresponding algebra $s^{(a, b)}=\mathbf{J}_{\mathbf{z}^{\prime}}^{(a, b)} \oplus \mathbf{J}_{\mathbf{z}^{\prime}}^{(a+b, 0)}$. Then we have:

$$
s^{(a, b)}=\mathbf{J}_{\mathbf{z}^{\prime}}^{(a, 0)} \oplus \mathbf{J}_{\mathbf{z}^{\prime}}^{(0, b)} \simeq \mathbf{J}_{\mathbf{z}^{\prime}} \oplus \mathbf{J}_{\mathbf{z}^{\prime}}
$$

I. e. $s^{\prime(a, b)}$ can be considered as a representation of the Lie algebra $\mathbf{J}_{z^{\prime}} \oplus$ $\mathbf{J}_{z^{\prime}}$. Let $\chi$ be the character of the corresponding group representation, belonging to the Lie algebra representation $\mathbf{J}_{\mathbf{z}^{\prime}}$. Then the character of the group representation belonging to the Lie algebra representation $s^{(a, b)}$ is $\chi^{(a, b)}\left(g_{1}, g_{2}\right)=a \chi\left(g_{1}\right)+b \chi\left(g_{2}\right)$. Therefore the representations $s^{(a, b)}$ and $s^{\prime\left(a^{\prime}, b^{\prime}\right)}$ are inequivalent unless $(a, b)=\left(a^{\prime}, b^{\prime}\right)$ up to an order. This proves the rest part of the Theorem. In fact, by Proposition 1.1, if $N_{J}^{(a, b)}$ and $N_{J}^{\left(a^{\prime}, b^{\prime}\right)}$ are isometric, the spaces $\mathbf{J}_{\mathbf{z}}^{(a, b)}$ and $\mathbf{J}_{\mathbf{z}}^{\left(a^{\prime}, b^{\prime}\right)}$ (and consequently also the Lie algebras $s^{(a, b)}$ and $\left.s^{\left(a^{\prime}, b^{\prime}\right)}\right)$ are conjugate (see (2.4)).

If $\mathbf{J}_{\mathbf{z}}$ is not a Lie algebra but it contains a non-Abelian Lie subalgebra, apply the above considerations to a maximal non-Abelian Lie subalgebra in order to have a proof also in these more general cases.

A different proof of this Statement can be given by means of Proposition 5.4 , where we prove that the Riemannian curvatures on $\sigma$-equivalent spaces are just isotonal in general.

Remark 2.2 The proof of the above Theorem breaks down if $\mathbf{J}_{\mathbf{z}}$ is just a linear space and it is not closed with respect to the Lie bracket (commutator) of endomorphisms. The following example shows that the above Theorem can not be generalized to such general cases straightforwardly.

In this example $\mathbf{z}=\mathbf{R}^{3}$, where $\mathbf{R}^{3}$ is considered as the space of imaginary quaternions. The endomorphisms $\mathbf{J}_{Z}$ acting on the quaternionic space $\mathbf{H}=$ $\mathbf{R}^{4}$ are defined by

$$
\mathbf{J}_{Z}(Q)=(L+R)_{Z}(Q)=Z Q+Q Z
$$

(It should be mentioned, that the Lie algebra $\left\{\mathbf{n}=\mathbf{R}^{4} \oplus \mathbf{R}^{3},\langle\rangle,, \mathbf{J}=R+L\right\}$ was introduced first in [GW3].) 
Since

$$
\left[(L+R)_{Z_{1}},(L+R)_{Z_{2}}\right]=(L-R)_{Z_{1} Z_{2}-Z_{2} Z_{1}}
$$

the space $(L+R)_{\mathbf{z}}$ is not closed with respect to the Lie bracket of endomorphisms. Furthermore, for the conjugation $K(Q)=\bar{Q}$ we have:

$$
K(L+R)_{Z} K^{-1}(Q)=\overline{Z \bar{Q}+\bar{Q} Z}=-(L+R)_{Z}(Q) .
$$

This means, that for any pair $(a, b)$ of natural numbers the orthogonal transformations $C=i d$ and

$$
A\left(X_{1}, \ldots, X_{a+b}\right)=\left(X_{1}, \ldots, X_{a}, \bar{X}_{a+1}, \ldots, \bar{X}_{a+b}\right)
$$

establish an isometry between $N_{J}^{(a, b)}$ and $N_{J}^{(a+b, 0)}$.

Remark 2.3 $\sigma$-equivalent spaces have inequivalent Riemannian curvature in general. For instance, in [Sz3] we prove (cf. formulas (1.17)-(1.20)) that the spaces $\mathbf{H}_{3}^{(a, b)}$ are distinguished by the eigenvalues of the curvature operator $R_{a b}{ }^{c d}$. (See more about this problem in $\S 5$.)

However, by (1.8), the $\sigma^{(a+b)}$-equivalent spaces have identical Ricci curvature at the origin, providing new examples for non-isomorphic Lie groups with locally non-isometric invariant metrics yet having equivalent Ricci curvature.

The reader can find such examples in [Ka]. The main difference between these two type of examples is that in Karidi's examples the spaces have different degrees of the balls' volume growth, while in our examples the spaces have the same Riemannian density function on polar coordinate neighborhood around the origin (this latter statement will be proven in a subsequent paper).

\section{3. $\S$ Technicalities on Ball $\times$ Torus- and on Ball-type domains}

The spectrum coincidences will be considered on the following two types of manifolds:

(1) Let $\Gamma$ be a full lattice on the Z-space spanned by a basis $\left\{e_{1}, \ldots, e_{l}\right\}$. For an $l$-tuple $\alpha=\left(\alpha_{1}, \ldots, \alpha_{l}\right)$ of integers the corresponding lattice point is $Z_{\alpha}=\alpha_{1} e_{1}+\cdots+\alpha_{l} e_{l}$. Since $\Gamma$ is a discrete subgroup, one can consider the factor manifold $\Gamma \backslash N$ with the factor metric. This factor manifold is 
a principal fiber bundle with the base space $\mathbf{v}$ and with the fiber $T_{X}$ at a point $X \in \mathbf{v}$. Each fiber $T_{X}$ is naturally identified with the torus $T=\Gamma \backslash \mathbf{z}$. The projection $\pi: \Gamma \backslash N \rightarrow \mathbf{v}$ defined by $\pi: T_{X} \rightarrow X$ projects the inner product from the horizontal subspace (= orthogonal complement to the fibers) to the euclidean inner product $\langle$,$\rangle on the X-space.$

Consider also a euclidean ball $B_{\delta}$ of radius $\delta$ around the origin of the Xspace and restrict the fiber bundle onto $B_{\delta}$. Then this fiber bundle $\left(B_{\delta}, T\right)$ has the boundary $\left(S_{\delta}, T\right)$, which is also a principal fiber bundle over the sphere $S_{\delta}$.

When we consider the spectrum problems on these manifolds, we call these cases Ball $\times$ Torus- and Sphere $\times$ Torus-cases.

Only these manifolds with the Laplacian spectrum on functions were investigated in the literature so far.

(2)In this paper we consider also such domains around the origin which are homeomorphic to a $(k+l)$-dimensional ball, and their smooth boundaries can be described as level sets by equations of the form $f(|X|, Z)=0$. In this case the boundary is homeomorphic to a sphere $S^{k+l-1}$ such that the boundary points form a euclidean sphere of radius $\delta(Z)$, for any fixed Z. I. e. the boundary can be described by the equation $|X|^{2}-\delta^{2}(Z)=0$. We call these cases Ball-cases resp. Sphere-cases.

In the following we need also the explicit form of the normal vector $\boldsymbol{\mu}$ at the boundary of these domains. From the equation

$$
\nabla f=g r a d f=\sum_{i} \mathbf{X}_{i}(f) \mathbf{X}_{i}+\sum_{\alpha} \mathbf{Z}_{\alpha}(f) \mathbf{Z}_{\alpha}
$$

we get (by using the special function $f(|X|, Z)=|X|^{2}-\delta^{2}(Z)$ ) that this unit normal vector at a point $(X, Z)$ is

$$
\boldsymbol{\mu}=\left(4|X|^{2}+\frac{1}{4}\left|\mathbf{J}_{\nabla \delta^{2}}(X)\right|^{2}+\left|\nabla \delta^{2}\right|^{2}\right)^{-\frac{1}{2}}\left(2 X-\frac{1}{2} \mathbf{J}_{\nabla \delta^{2}}(X)-\nabla \delta^{2}\right)
$$

where $\boldsymbol{\mu}$ is considered as an element of the Lie algebra. Notice that in Case (1) the $\boldsymbol{\mu}$ has the simple form $\boldsymbol{\mu}=X /|X|$.

It can be written in the following regular vector form (cf. (1.2)):

$$
\boldsymbol{\mu}=A\left(2|X| E_{0}-\sum_{\alpha=1}^{l}\left(\partial_{\alpha} \delta^{2}\right)\left(\frac{1}{2}|X| E_{\alpha}+\sum_{\beta=1}^{l}\left(1+\frac{1}{4}\left\langle\mathbf{J}_{\alpha}(X), \mathbf{J}_{\beta}(X)\right\rangle\right) e_{\beta}\right)\right)
$$


where $\left\{e_{1}, \ldots, e_{l}\right\}$ is an orthonormal basis of $\mathbf{z}$ and $E_{\alpha}=\mathbf{J}_{\alpha}\left(E_{0}\right) ; E_{0}=$ $X /|X|$ furthermore

$$
A=\left(4|X|^{2}+\frac{1}{4}\left|\mathbf{J}_{\nabla \delta^{2}}(X)\right|^{2}+\left|\nabla \delta^{2}\right|^{2}\right)^{-\frac{1}{2}}
$$

In the literature this is the first paper which involves also the manifolds of type (2) into the spectral investigations. The considerations require completely different techniques on these two types of manifolds.

Since $\left(\mathbf{J}_{Z}^{\sigma}\right)^{2}=\mathbf{J}_{Z}^{2}$, the endomorphisms $\mathbf{J}_{Z}$ and $\mathbf{J}_{Z}^{\sigma}$ are isospectral (conjugate). Therefore the following Statement immediately follows from the Gordon-Wilson Theorem [GW3]. However, we describe a proof here which is a straightforward adoption of the proof given on the special spaces $\mathbf{H}_{3}^{(a, b)}$ in [Sz3]. The main difference between this proof and the proof given in [GW3] is that our proof does not use the total geodesic assumption on the tori of the considered torus bundle. This idea was used also in [GS].

Theorem 3.1. The metrics on $\sigma$-equivalent spaces are Dirichlet and Neumann isospectral on any of the manifolds described in the Ball $\times$ Torus-cases.

Proof. First consider the Weierstrass decomposition

$$
L^{2}(\Gamma \backslash N)=\oplus W^{(\alpha)}
$$

of the $L^{2}$ function space, where $W^{(\alpha)}$ consists of functions of the form

$$
\phi^{(\alpha)}(X, Z)=\varphi(X) e^{2 \pi \mathbf{i}\left\langle Z_{\alpha}, Z\right\rangle}
$$

for any fixed lattice point $Z_{\alpha}$.

By (1.6), any of the spaces $W^{\alpha}$ is invariant under the action of the Laplacian. More precisely we have:

$$
\Delta \phi^{(\alpha)}=\left(\square_{(\alpha)} \varphi\right) e^{2 \pi \mathbf{i}\left\langle Z_{\alpha}, Z\right\rangle},
$$

where

$$
\square_{(\alpha)}=\Delta_{X}+2 \pi \mathbf{i} D_{(\alpha)} \bullet-4 \pi^{2}\left(\left|Z_{\alpha}\right|^{2}+\frac{1}{4}\left|\mathbf{J}_{Z_{\alpha}}(X)\right|^{2}\right) .
$$

Next we construct intertwining operator, $\tau_{*}$, which intertwines the corresponding Laplacians. 
Since $\mathbf{J}_{Z_{\alpha}}^{\sigma}$ and $\mathbf{J}_{Z_{\alpha}}$ are isospectral (conjugate), there exists an orthogonal transformation $\tau^{(\alpha)}$ on the X-space such that

$$
\mathbf{J}_{Z_{\alpha}}^{\sigma}=\tau^{(\alpha)} \circ \mathbf{J}_{Z_{\alpha}} \circ \tau^{(\alpha)-1} .
$$

(In [Sz3], this transformation $\tau^{(\alpha)}$ is individually constructed, using conjugations and products by $Z_{\alpha}$.) Therefore we have

$$
D_{\alpha}^{\sigma} \bullet=\tau_{*}^{(\alpha)} D_{\alpha} \bullet \tau^{(\alpha) *} \quad ; \quad \square_{(\alpha)}^{\sigma}=\tau_{*}^{(\alpha)} \square_{(\alpha)} \tau^{(\alpha) *} .
$$

Thus the operator $\tau_{*}=\oplus \tau_{*}^{(\alpha)}$ intertwines the Laplacians $\Delta^{\sigma}$ and $\Delta$ on the whole $L^{2}$ function spaces.

It is plain that $\tau_{*}$ does not change the Dirichlet boundary conditions. By (3.1), the normal vector is $\boldsymbol{\mu}=X /|X|$ at a boundary point $(X, Z)$. Since the orthogonal transformations leave the differentiations from the radial directions invariant, also the Neumann boundary condition is invariant by the action of $\tau_{*}$. This proves the Statement completely.

The above applied method of Fourier transform breaks down in the Ballcase, since the functions of the form (3.5) do not satisfy neither the Dirichlet nor the Neumann boundary conditions. For constructing an appropriate intertwining operator in the Ball-cases, we have to develop a completely different technique.

Remark 3.2 Any 2-step nilpotent Lie group $N$ can be extended into a solvable group $S N$ defined on the half space $\mathbf{n} \times \mathbf{R}_{+}$with multiplication given by

$$
(X, Z, t)\left(X^{\prime}, Z^{\prime}, t^{\prime}\right)=\left(X+t^{\frac{1}{2}} X^{\prime}, Z+t Z^{\prime}+\frac{1}{2} t^{\frac{1}{2}}\left[X, X^{\prime}\right], t t^{\prime}\right) .
$$

This group has the Lie algebra $\mathbf{s}=\mathbf{n} \oplus \mathbf{t}$ with the Lie bracket satisfying

$$
\left[\partial_{t}, X\right]=\frac{1}{2} X \quad ; \quad\left[\partial_{t}, Z\right]=Z \quad ; \quad[\mathbf{n}, \mathbf{n}]_{/ S N}=[\mathbf{n}, \mathbf{n}]_{/ N},
$$

for any $X \in \mathbf{v}$ and $Z \in \mathbf{z}$. Consider on this Lie algebra the natural inner product $\langle$,$\rangle such that we keep the inner product on \mathbf{n}$ furthermore $\partial_{t} \perp \mathbf{n}$ and $\left|\partial_{t}\right|=1$. The left invariant extension of this inner product is denoted by $g$.

The explicit computations of the several geometric objects along with other technicalities on these solvable extensions are established in [Sz4]. For 
instance, we prove there that two appropriate solvable extensions are isometric iff the corresponding nilpotent groups are isometric (cf. Proposition 1.2 in [Sz4]; this Statement is established also in [GW1]). The Laplacian acting on functions has the following explicit form (cf. (1.12) in [Sz4]):

$$
\begin{aligned}
\Delta= & t \Delta_{X}+t^{\frac{1}{2}} \Delta_{Z}+\frac{1}{4} t \sum_{\alpha ; \beta=1}^{l}\left\langle\mathbf{J}_{\alpha}(X), \mathbf{J}_{\beta}(X)\right\rangle \partial_{\alpha \beta}^{2} \\
& +t \sum_{\alpha=1}^{l} \partial_{\alpha} D_{\alpha} \bullet+t^{2} \partial_{t}^{2}+\left(1-\frac{k}{2}-l\right) t \partial_{t} .
\end{aligned}
$$

(This formula is established also in [GS].)

The above spectral investigations can be extended onto these solvable groups on the following domains.

The Solvable Ball $\times$ Torus Cases: A group $S N$ can be considered as a principal fiber bundle (vector bundle) over the $(X, t)$-space, fibered by the $\mathrm{Z}$-space. Again, $\Gamma$ is a full lattice on the $\mathrm{Z}$-space, while $D_{R(t)}$ is a domain on the $(X, t)$-space diffeomorphic to a $(k+1)$-dimensional ball whose smooth boundary can be described by an equation of the form $|X|=R(t)$. Then consider the torus bundle $\left(\Gamma / \mathbf{z}, D_{R(t)}\right)$ over $D_{R(t)}$. The normal vector $\boldsymbol{\mu}$ at a boundary point $(X, Z, t)$ is of the form $\boldsymbol{\mu}=A(t) X+B(t) \partial_{t}$, where $A(t)$ and $B(t)$ are determined by $R(t)$.

The Solvable Ball Case: In this case we consider a domain $D$ diffeomorphic to a $(k+l+1)$-dimensional ball whose smooth boundary (diffeomorphic to $\left.S^{k+l}\right)$ can be described as a level surface in the form $|X|=R(Z, t)$. The normal vector $\boldsymbol{\mu}$ at a boundary point $(X, Z, t)$ has the form

$$
\begin{gathered}
\boldsymbol{\mu}=A_{0}(|X|, Z, t) E_{0}+C(Z, t) \partial_{t}+ \\
\sum_{i=1}^{l}\left(A_{i}(|X|, Z, t) E_{i}+B_{i}(|X|, Z, t) e_{i}\right),
\end{gathered}
$$

where the coefficient functions $A_{\alpha} B_{i}$ and $C$ are determined by $R(Z, t)$ (cf. (3.24)-(3.26) in $[\mathrm{Sz} 4])$.

Then, by repeating the very same arguments used in the nilpotent cases we get (the only modification needed is that the function $\varphi$, in (3.5), should have the form $\varphi(X, t))$

Theorem 3.3. The metrics on $\sigma$-equivalent solvable spaces are Dirichlet and Neumann isospectral on any of the domains described in the Solvable Ball $\times$ Torus cases. 
Remark 3.4 Finally, we check that how these ideas are (or, are not) working in the case of forms.

In the Ball $\times$ Torus-cases, the Fourier decomposition on the $L^{2}$ space of $\mathrm{r}$-forms is

$$
L_{r}^{2}(\Gamma / N)=\oplus W_{r}^{\alpha}
$$

where $W_{r}^{\alpha}$ is spanned by the r-forms of the form

$$
\Omega^{(\alpha)}=\varphi(X) e^{2 \pi \mathbf{i}\left\langle Z_{\alpha}, Z\right\rangle} \omega .
$$

Here $\varphi(X)$ is an $L^{2}$-function and $\omega$ is an invariant(!) r-form.

The invariant $r$-forms can be expressed by the invariant 1 -forms

$$
\begin{gathered}
\theta_{i}(.)=g\left(\mathbf{X}_{i}, .\right)=d x^{i}(.) \\
\vartheta_{\beta}(.)=g\left(\mathbf{Z}_{\beta}, .\right)=d z_{\beta}(.)-\frac{1}{2} \sum_{i=1}^{l}\left\langle\mathbf{J}_{\beta}(X), \partial_{i}\right\rangle d x_{i}(.) .
\end{gathered}
$$

For computing the right sides of these formulas we used (1.1) and (1.11). From these formulas we immediately get that the forms $\theta_{i}$ are closed, while

$$
d \vartheta_{\beta}=-\frac{1}{2} \mathbf{J}_{\beta}^{j i} \theta_{j} \wedge \theta_{i}
$$

Actually this non-closedness of the forms $\vartheta_{\beta}$ "kills" the method of the Fourier transform if we try to prove the isospectral property of $\sigma$-equivalent spaces on the spectra of forms of degree $\geq 1$. In fact, in case of functions the method of Fourier transform reduces the spectral investigation to a case when only a single endomorphism $\mathbf{J}_{\alpha}$ is involved. The above equation shows that such kind of reduction is not possible in case of higher order forms. (Check $\S 5$. to see that the same problem emerges also with respect to the $\delta$-operator.)

\section{4. $\oint$ Constructing Isospectral Balls on Spaces with Anticommutators}

The final goal of this section is to find such/those particular $\sigma$-deformations providing isospectral metrics also on the Ball- and Sphere-type manifolds. It turns out that this goal can be achieved on spaces having particular Endomorphism Spaces described as follows. 
If we perform $\sigma$-deformations only on a particular subspace of the Endomorphism Space $\mathbf{J}_{\mathbf{z}}$ and we keep the endomorphisms belonging to the orthogonal complement unchanged, such a deformation is said to be partial $\sigma$-deformation or P $\sigma$-deformation. More precisely, if the deformation is performed on the endomorphisms belonging to a subspace $\mathbf{s} \subset \mathbf{z}$, the deformation is called $\sigma_{\mathrm{s}}$-deformation and if $\sigma$ has the form $\sigma=(i d,-i d)$ with respect to a decomposition $\mathbf{v}=\mathbf{v}^{(a)} \oplus \mathbf{v}^{(b)}$, it is called $\sigma_{\mathbf{s}}^{(a+b)}$-deformation.

If all the invariant subspaces of the irreducible decomposition of the $\mathrm{X}$ space with respect to the action of $\mathbf{J}_{\mathbf{z}}$ have dimension greater than 2 , then a $\sigma_{\mathbf{s}}$-deformation is a $\sigma_{\mathbf{s}}^{(a+b)}$-deformation. For the sake of the simplicity, we suppose that the Endomorphism Spaces satisfy this non-degenerating property. By this assumption only trivial cases are excluded (these cases are really trivial when The Endomorphism Space contains an anticommutator).

These deformations define a much wider class then the $\sigma$-deformations. However, a $P \sigma$-deformation may be equivalent to a $\sigma$-deformation. In the following we prove that a $\sigma$-deformation is equivalent to a $\sigma_{\mathrm{A}}$-deformation if the endomorphism space $\mathbf{J}_{\mathbf{z}}$ contains a so called anticommutator $A$. (Here A denotes the 1-dimensional subspace spanned by $A$.) It turns out that these particular $\sigma \simeq \sigma_{\mathbf{A}^{-}}$-deformations are the desired ones and they provide isospectral metrics also on the Ball- and Sphere-type manifolds and not only on the Ball $\times$ Torus- and the Sphere $\times$ Torus-type manifolds. However, it is still an open question, whether they provide all the solutions for the problem.

We establish all these Theorems in 3 Steps.

\section{Endomorphism Spaces with Anticommutators (alias $E S W_{A}$ )}

A non-degenerated endomorphism $A=\mathbf{J}_{Z}$ is said to be an anticommutator in $\mathbf{J}_{\mathbf{z}}$ if $A \circ B=-B \circ A$ holds for all $B \in \mathbf{J}_{Z^{\perp}}$, i. e., if the $A$ anticommutes with all of the orthogonal endomorphisms. An anticommutator satisfying $A^{2}=-i d$ is said to be a unit anticommutator.

Any anticommutator can be written in the form $A=S \circ A_{0}$, where $A_{0}$ is a unit endomorphism anticommuting with the elements of $\mathbf{A}^{\perp}$ and the symmetric scaling endomorphism $S$ (which is one of the square root of $-A^{2}$ ) is commuting with all elements of the $E S W_{A}$.

An orthogonal transformations $\sigma$, used for defining $\sigma$-deformations on a non-degenerated Endomorphism Space, is always of the form $\sigma=(i d,-i d)$ with respect to an appropriate decomposition $\mathbf{v}=\mathbf{v}^{(a)} \oplus \mathbf{v}^{(b)}$. In these cases, the $\sigma$-deformation of an endomorphism $\mathbf{J}_{Z}$ is denoted by $\mathbf{J}_{Z}^{(a, b)}$ and 
the original endomorphism can be denoted also by $\mathbf{J}_{Z}^{(a+b, 0)}$.

Since the equation $\mathbf{J}_{Z} \mathbf{J}_{Z^{*}}+\mathbf{J}_{Z^{*}} \mathbf{J}_{Z}=-2\left\langle Z, Z^{*}\right\rangle i d$ holds on any Heisenberg type group (cf. (1.4) in [CDKR]), all the endomorphisms are anticommutators in the Endomorphism Spaces belonging to these groups.

In this paper we consider such Endomorphism Spaces where the anticommuting elements form at least a 1-dimensional subspace. The complete description of the Endomorphism Spaces having non-trivial anticommuting elements is given in [Sz4] (cf. Canonical Matrix Representation Theorem 2.1 of the $E S W_{A}$ 's). Here we introduce only the Quaternionic $E S W_{A}$ 's, which show the wide range of such spaces.

Let $A$ be a $k \times k$ diagonal matrix such that it has the same imaginary quaternionic number a in the main diagonal. Consider also a linear space $\mathbf{J}_{A}^{\perp}$ of $k \times k$ symmetric matrices such that the entries are imaginary quaternions perpendicular to a. All these matrices define a skew-symmetric real matrix-map on $\mathbf{H}^{k}=\mathbf{R}^{4 k}$. (In this matrix transformation we multiply by the entries of the matrix always on the same side, i. e. we can choose between left or right representations of these matrices.) Then the endomorphism $\mathbf{J}_{A}$ is an anticommutator in the endomorphism space $\mathbf{J}_{A}^{\perp} \oplus \mathbf{A}$. Also the Cartesian products of such spaces are endomorphism spaces with non-trivial anticommutators.

Surprisingly enough, if an endomorphism space has an anticommutator $A$, a $\sigma$-deformation is equivalent to the $\sigma_{\mathbf{A}}$-deformation. More precisely we have

Reduction Theorem 4.1. If $\mathbf{J}_{\mathbf{s}}$ is an odd-dimensional subspace of the anticommutators in a non-degenerated $E S W_{A}$, then a $\sigma=\sigma^{(a+b)}$-deformation is equivalent to the partial $\sigma_{\mathrm{s}}=\sigma_{\mathrm{s}}^{(a+b)}$-deformation, $i$. e. the corresponding metrics are isometric.

If the space $\mathbf{s}$ is even dimensional, then $a \sigma_{\mathbf{s}}=\sigma_{\mathbf{s}}^{(a+b)}$-deformation provides a space which is isometric to the original space.

Proof. Let $\mathbf{J}_{\mathbf{z}}$ be a linear space of endomorphisms with a non-degenerated anticommutator $\mathbf{J}_{A}$. By an appropriate rescaling of the maximal invariant spaces of $\mathbf{J}_{A}$, one can find an endomorphism $A_{0}$ (which may not be in $\mathbf{J}_{\mathbf{z}}$ ) such that $A_{0}^{2}=-i d$. Since all the endomorphisms $B \in \mathbf{J}_{A^{\perp}}$ leave the maximal eigensubspaces of $A$ invariant, this $A_{0}$ is commuting with $A$ and it is anticommuting with the endomorphisms from the orthogonal space $J_{A^{\perp}}$.

Let $\left\{J_{A_{1}}, \ldots, J_{A_{r}}\right\}$ be an orthonormal basis on the subspace $J_{\mathbf{s}}$. The orthogonal transformation $A_{i 0}^{(b)}$ on the space $\mathbf{v}=\mathbf{v}^{(a)} \oplus \mathbf{v}^{(b)}$ is defined by 
$i d \times A_{i 0 / \mathbf{v}^{(b)}}$. Then the orthogonal transformation $A_{0}^{(b)}=A_{10}^{(b)} \circ \cdots \circ A_{r 0}^{(b)}$ and $C=i d$ establish the isometries (cf. Proposition 1.1) between the corresponding spaces.

In fact, if $r=2 m+1$, we have

$$
\left(A_{0}^{(b)}\right)^{-1} \mathbf{J}_{Z}^{(a+b, 0)} A_{0}^{(b)}=\mathbf{J}_{Z}^{(a, b)}, \forall Z \in \mathbf{s}^{\perp} \quad ; \quad\left(A_{0}^{(b)}\right)^{-1} \mathbf{J}_{A_{i}}^{(a, b)} A_{0}^{(b)}=\mathbf{J}_{A_{i}}^{(a, b)} .
$$

If $r=2 m$, then we get

$$
\left(A_{0}^{(b)}\right)^{-1} \mathbf{J}_{Z}^{(a+b, 0)} A_{0}^{(b)}=\mathbf{J}_{Z}^{(a+b, 0)}, \forall Z \in \mathbf{s}^{\perp} \quad ; \quad\left(A_{0}^{(b)}\right)^{-1} \mathbf{J}_{A_{i}}^{(a, b)} A_{0}^{(b)}=\mathbf{J}_{A_{i}}^{(a+b, 0)},
$$

which prove the statement completely.

In the following we consider an endomorphism space $J_{\mathbf{z}}$ with an anticommutator $J_{A}$. For the sake of the simplicity we suppose that $J_{A}^{2}=-i d$, i. e. it is a unit anticommutator. All of the theorems proven in the following can be established also for general anticommutators. In the end we describe all the modifications what should be done for having the proofs in these general cases.

The great advantage on the $E S W_{A}$ 's is that a $\sigma$-deformation can be expressed as a $\sigma_{\mathbf{A}}$-deformation and an intertwining operator for the corresponding Isospectrality Theorem can be established by means of the endomorphism $A$.

\section{Harmonic Analysis based on a Unit Anticommutator}

The main tool in this paper is a sort of Harmonic Analysis established by means of a unit anticommutator. We define a certain decomposition of the $L^{2}$ function space with the help of this unit endomorphism and then we use this decomposition for constructing appropriate intertwining operators.

For a fixed $\mathrm{X}$-vector $Q$, we define the complex valued function

$$
\Theta_{Q}(X)=\left\langle Q+\mathbf{i} \mathbf{J}_{A}(Q), X\right\rangle .
$$

Then the complex valued function space is spanned by the functions of the form

$$
\Phi_{\left(Q_{i}, p_{i}, Q_{i}^{*}, p_{i}^{*}, \varphi\right)}(X, Z)=\left(\Pi_{i=1}^{r} \Theta_{Q_{i}}^{p_{i}}(X) \bar{\Theta}_{Q_{i}^{*}}^{p^{*}}(X)\right) \varphi(Z)
$$

Notice, that the functions of the pure form

$$
\Phi_{\left(Q_{i}, p_{i}\right)}(X)=\Pi_{i=1}^{r} \Theta_{Q_{i}}^{p_{i}}(X) \quad ; \quad \bar{\Phi}_{\left(Q_{i}, p_{i}\right)}(X)=\Pi_{i=1}^{r} \bar{\Theta}_{Q_{i}}^{p_{i}}(X)
$$


are harmonic with respect to the euclidean Laplacian $\Delta_{X}$ on the $X$-space. However, the polynomials of the mixed form are not harmonic, since

$$
\Delta_{X} \Theta_{Q}(X) \bar{Q}_{Q^{*}}(X)=2\left\langle Q, Q^{*}\right\rangle+2 \mathbf{i}\left\langle\mathbf{J}_{A}^{(a, b)}(Q), Q^{*}\right\rangle .
$$

In the following we find more specific functions which still span the $L^{2}$ function space on the X-space. First we rewrite the functions (4.2) in the form

$$
\varphi(|X|, Z) \Phi_{\left(Q_{i}, p_{i}, Q_{i}^{*}, p_{i}^{*}\right)}\left(X_{u}\right),
$$

where $X_{u}=X /|X|$ is the unit radial $\mathrm{X}$-vector.

Notice that the $q^{\text {th }}$ order polynomials

$$
\begin{aligned}
& \Phi_{\left(Q_{u}, q\right)}(X)=\left\langle Q_{u}+\mathbf{i} \mathbf{J}_{A}\left(Q_{u}\right), X\right\rangle^{q}, \\
& \bar{\Phi}_{\left(Q_{u}, q\right)}(X)=\left\langle Q_{u}-\mathbf{i} \mathbf{J}_{A}\left(Q_{u}\right), X\right\rangle^{q}
\end{aligned}
$$

are harmonic on the euclidean $\mathrm{X}$-space whose restrictions onto the unit sphere $S$ (around the origin of the X-space) define $q^{t h}$ order eigenfuctions of the Laplacian $\Delta_{S}$. Let us note that the whole space $\mathbf{H}^{(q)}$ of $q^{\text {th }}$ order eigenfunctions is not spanned by these functions. The "missing" functions can be furnished with the help of the $q^{\text {th }}$ order functions of the form $\Phi_{\left(Q_{i}, p_{i}, Q_{i}^{*}, p_{i}^{*}\right)}\left(X_{u}\right)$, where $q=\sum_{i} p_{i}+\sum_{i} p_{i}^{*}=s+(q-s)$, as follows

Such a function is not an eigenfunction of the Laplacian $\Delta_{S}$, however, it is an eigenfunction of the differential operator $D_{A} \bullet$ with the eigenvalue $(2 s-q)$ i. By orthogonal projection, project all these functions onto the function space $\mathbf{H}^{(q)}$. The image space is denoted by $\mathbf{H}^{(s, q-s)}$.

In [Sz3] we proved that the operators $\Delta_{S}$ and $D_{A} \bullet$ commute, where $D_{A} \bullet$ means directional derivative with respect to the vector field $X \rightarrow \mathbf{J}_{A}(X)$. (This proof is based on the simple fact that the vector field $J_{A}(X)$ tangent to the $\mathrm{X}$-spheres around the origin is an infinitesimal generator of isometries on these spheres.) Therefore the whole space $\mathbf{H}^{(q)}$ is invariant under the action of $D_{A} \bullet$ and the subspace $\mathbf{H}^{(s, q-s)} \subset \mathbf{H}^{(q)}$ is an eigensubspace of this operator with the eigenvalue $(2 s-q)$ i. Thus the decomposition $\mathbf{H}^{(q)}=$ $\oplus_{s=0}^{q} \mathbf{H}^{(s, q-s)}$ is an orthogonal direct sum corresponding to the common eigensubspace decomposition of the two commuting differential operators $\Delta_{S}$ and $D_{A} \bullet$.

We need a more accurate description of the functions which span these eigensubspaces. For this description we use the kernel functions

$$
H_{(q)}\left(Q_{u}, Q_{u}^{*}\right)=\sum_{j=1}^{N_{q}} \eta_{j}^{(q)}\left(Q_{u}\right) \eta_{j}^{(q)}\left(Q_{u}^{*}\right),
$$


where $\left\{\eta_{1}^{(q)}, \ldots, \eta_{N_{q}}^{(q)}\right\}$ is an orthonormal basis on the subspace $\mathbf{H}^{(q)}$. In [Be] it is proven (cf. Lemma 6.94) that for any fixed $Q_{u}$, the eigen function $H_{(q)}\left(Q_{u},.\right) \in \mathbf{H}^{(q)}$ is a radial one (i.e. it has the form $C_{q}\left\langle Q_{u}, .\right\rangle^{q}+\cdots+$ $\left.C_{1}\left\langle Q_{u},.\right\rangle+C_{0}\right)$ with $H_{(q)}\left(Q_{u}, Q_{u}\right)=1$. (One can find appropriate reference to this Statement also in [Sz1] $(\S 2$, Pages 8-11) where this kernel is scrutinized on arbitrary harmonic spaces.) These kernel functions can be used for projecting the functions into the subspaces $\mathbf{H}^{(q)}$. More precisely, for any function $\Psi \in L^{2}(S)$ we have

$$
\Psi\left(Q_{u}\right)=\sum_{q=0}^{\infty} \int H_{(q)}\left(Q_{u}, Q_{u}^{*}\right) \Psi\left(Q_{u}^{*}\right) d Q_{u}^{*}=\sum h_{(q)}(\Psi)\left(Q_{u}\right),
$$

which is called the spherical decomposition of $\Psi$ by the spherical harmonics. The operators $h_{(q)}: L^{2}(S) \rightarrow \mathbf{H}^{(q)}$ project the $L^{2}$ function space to the corresponding eigensubspace of the Laplacian.

One of the most important properties of these operators is the commutativity with the differential operators $D_{\alpha} \bullet$. This commutativity immediately follows from the fact that the $\mathbf{J}_{\alpha}(X)$ is an infinitesimal generator of one parametric families of isometries on the euclidean sphere $S$, furthermore, the projections $h_{(q)}$ are invariant with respect to these isometries.

For an $\mathrm{X}$-vector $Q$, we define the complex vector

$$
\mathbf{Q}=Q+\mathbf{i} \mathbf{J}_{A}(Q) \text {. }
$$

Then, by substituting $Q_{u}=1 / 2\left(\mathbf{Q}_{u}+\overline{\mathbf{Q}}_{u}\right)$ into the above expression of the radial kernel $H_{(q)}\left(Q_{u},.\right)$, we get

Proposition 4.2. The $r^{\text {th }}$ order polynomial space $\mathbf{P}^{(r)}$ is the direct sum of the subspaces $\mathbf{P}^{(p, r-p)}$ spanned by the polynomials of the form $\Theta_{Q}^{p} \bar{\Theta}_{Q}^{r-p}$, where $Q \in \mathbf{v}$ and $0 \leq p \leq r$. The space $\mathbf{P}^{(p, r-p)}$ consists of the $r^{\text {th }}$ order eigen polynomials of the differential operator $D_{A} \bullet$ with eigenvalue $(r-p) \mathbf{i}$.

The projection $h_{(r)}$ establishes a one to one and onto map between the polynomial spaces $\mathbf{P}_{S}^{(p, r-p)}$ and $\mathbf{H}^{(p, r-p)}$. We denote this restricted map by $h_{/(r)}: \mathbf{P}_{S}^{(r)} \rightarrow \mathbf{H}^{(r)}$. The direct sum $T=\oplus_{r} h_{/(r)}$ of these maps defines an invertible operator on the whole function space $L_{S}^{2}$. This operator $T$ is commuting with the differential operators $D_{\alpha} \bullet$.

Above $\mathbf{P}_{S}^{(p, r-p)}$ denotes the space of the corresponding restricted functions on the sphere $S$. This space is spanned by the functions of the form 
$\Theta_{Q_{u}}^{p} \bar{\Theta}_{Q_{u}}^{r-p}$, where $Q_{u}$ is a unit vector. Let us mentioned that the projected functions $h_{/(r)}\left(\Theta_{Q_{u}}^{p} \bar{\Theta}_{Q_{u}}^{r-p}\right)$ can be written in the following form

$$
h_{/(r)}\left(\Theta_{Q}^{p} \bar{\Theta}_{Q}^{r-p}\right)=\sum_{s=0}^{\min (p, r-p)} A_{s} \Theta_{Q}^{p-s} \bar{\Theta}_{Q}^{r-p-s},
$$

where $A_{0}=1$ furthermore the higher order coefficients can be computed by the recursive formula

$$
2|Q|^{2}(p-s)(r-p-s) A_{s}+(s+1)(\operatorname{dim}(\mathbf{v})+r-2) A_{s+1}=0 .
$$

This statement follows from the fact that the homogeneous polynomial $\sum_{s} A_{s}\langle X, X\rangle^{s} \Theta_{Q}^{p-s} \bar{\Theta}_{Q}^{r-p-s}$ is harmonic with respect to the Laplacian $\Delta_{X}$ and therefore its restriction onto $S$ is an eigenfunction of the Laplacian $\Delta_{S}$. Actually this statement proves the stronger Proposition asserting that the projected functions onto the lower order subspaces have the form

$$
h_{(q)}\left(\Theta_{Q}^{p} \bar{\Theta}_{Q}^{r-p}\right)=\sum_{s=0}^{\min (p, q-p)} A_{q s} \Theta_{Q}^{p-s} \bar{\Theta}_{Q}^{q-p-s},
$$

where $q \leq r$ and they are trivial $(=0)$ on the higher order subspaces.

By (4.7), the radial eigenfunctions span the eigenspaces $\mathbf{H}^{(r)}$, therefore the above formulas completely describe the maps $h_{/(r)}$. However, the above concept can be easily generalized to the case when $h_{/(r)}\left(\Pi_{i} \Theta_{Q_{i}}^{p_{i}} \bar{\Theta}_{Q_{i}}^{r_{i}-p_{i}}\right)$ should be figured out, where $\sum r_{i}=r$. In this case we look for the harmonic homogeneous polynomial of the form

$$
\Pi_{i} \sum_{s_{i}} A_{s_{i}}\langle X, X\rangle^{s_{i}} \Theta_{i}^{p_{i}-s_{i}} \bar{\Theta}_{i}^{r_{i}-p_{i}-s_{i}}
$$

To compute clean recursion formulas in this general cases is tiresome and fortunately we do not need them in this paper. However, the recursive computation of the coefficients $A_{s_{i}}$ is clear. Arrange this latter function according to the $s$-lines (the 0 -line corresponds to the original function) and then let the Laplacian act on this function. First the coefficients $A_{1_{i}}$ can be computed by considering the action of the Laplacian on the 0 - and the 1-line. E. t. c., the computation should be continued till it is finished.

This Computational Technique will be used later in this section. 
These formulas together with $h_{/(r)} D_{\alpha} \bullet=D_{\alpha} \bullet h_{/(r)}$ completely prove the second part of the Proposition.

In the following we represent the functions $\psi \in \mathbf{H}^{(r)}$ in the form $\psi=$ $h_{/(r)}\left(\psi^{*}\right)$, where $\psi^{*} \in \mathbf{P}_{S}^{(r)}$. We use this tool to simplify the considerations.

On the whole ambient space, $\mathbf{n}$, the function space is spanned by the functions of the form

$$
F(X, Z)=\varphi(|X|, Z) h_{/(r)}\left(\Theta_{Q_{u}}^{p} \bar{\Theta}_{Q_{u}}^{r-p}\right)\left(X_{u}\right),
$$

where $X_{u}=X /|X|$ is a unit vector. The decomposition with respect to these functions is called spherical decomposition. If we cancel $h_{/(r)}$ in (4.9), the corresponding decomposition with respect to these functions is called polynomial decomposition. The operator $T$ transforms a polynomial decomposition to a spherical decomposition.

\section{Constructing the Intertwining Operator}

If $\mathbf{v}=\mathbf{v}^{(a)} \oplus \mathbf{v}^{(b)}$ is the decomposition corresponding to a $\sigma_{A^{-} \text {-deformation }}$ on a space with non-degenerated $E S W_{A}$, then the $\sigma_{A}$-deformed group is denoted by $N_{\mathbf{z}}^{(a, b)}$. The original group $N_{\mathbf{z}}$ can be denoted also by $N_{\mathbf{z}}^{(a+b, 0)}$. This general notation is consistent with the particular notation $\mathbf{H}_{3}^{(a, b)}$, however, the $\sigma^{(a+b)}$-deformation is represented now as $\sigma_{A}^{(a, b)}$-deformation. If $N=N_{\mathbf{z}}^{(a, b)}$ and $N^{\prime}=N_{\mathbf{z}}^{\left(a^{\prime}, b^{\prime}\right)}$, where $a+b=a^{\prime}+b^{\prime}$, are $\sigma_{A^{-} \text {-deformations of }}$ the very same group, then $N$ and $N^{\prime}$ are clearly $\sigma_{\mathrm{A}}$-equivalent spaces. Consider these groups, the Intertwining Operator $\kappa$ between the corresponding complex function spaces is defined by

$$
\begin{gathered}
\kappa: L^{2}\left(N_{\mathbf{z}}^{(a, b)}, \mathbf{C}\right) \rightarrow L^{2}\left(N_{\mathbf{z}}^{\left(a^{\prime}, b^{\prime}\right)}, \mathbf{C}\right), \\
\kappa: F(X, Z) \rightarrow F^{\prime}(X, Z)=\varphi(|X|, Z) h_{/(r)}^{\prime}\left(\Theta \prime p_{Q_{u}}{\overline{\Theta^{\prime}}}_{Q_{u}}^{r-p}\right)\left(X_{u}\right),
\end{gathered}
$$

where $F$ is defined above by means of $\mathbf{J}_{A}^{(a, b)}$ and the functions $\Theta^{\prime}$ are defined on $N^{\prime}$ by means of $\mathbf{J}_{A}^{\left(a^{\prime}, b^{\prime}\right)}$.

Notice that this map maps the function space $\mathbf{H}^{(r)}$ onto $\mathbf{H}^{\prime(r)}=\mathbf{H}^{(r)}$, however, it is defined by means of the map

$$
\kappa^{*}: \mathbf{P}^{(r)} \rightarrow \mathbf{P}^{\prime(r)} \quad, \quad \kappa^{*}: \Theta_{Q}^{p} \bar{\Theta}_{Q}^{r-p} \rightarrow \Theta_{Q}^{\prime p} \bar{\Theta}_{Q}^{\prime r-p}
$$

and of the projection $h_{(r)}$. Unlike $\kappa$, the map $\kappa^{*}$ can be easily handled. If $\left\{E_{1}, \ldots, E_{K}\right\}$ is a basis on the X-space then $\kappa^{*}\left(\Pi \Theta_{E_{i_{r}}} \Pi \bar{\Theta}_{E_{j_{r}}}\right)=$ 
$\Pi \Theta_{E_{i_{r}}}^{\prime} \Pi \bar{\Theta}_{E_{j_{r}}}^{\prime}$ and in general, for arbitrary vectors $Q_{m}$, we get

$$
\kappa^{*}\left(\Pi \Theta_{Q_{i_{r}}} \Pi \bar{\Theta}_{Q_{j_{r}}}\right)=\Pi \Theta_{Q_{i_{r}}}^{\prime} \Pi \bar{\Theta}_{Q_{j_{r}}}^{\prime} .
$$

The operators $\kappa$ and $\kappa^{*}$ are connected by the equation $\kappa=T^{\prime} \circ \kappa^{*} \circ T^{-1}$. In the following we show the intertwining property of the map $\kappa$.

Consider the explicit expression (1.6) of the Laplacian $\Delta$. Since $\kappa$ : $\mathbf{H}^{(q)} \rightarrow \mathbf{H}^{\prime(q)}=\mathbf{H}^{(q)}$, the terms $\Delta_{X}+\Delta_{Z}$ and $\Delta_{X}^{\prime}+\Delta_{Z}^{\prime}$ are intertwined by this map. In fact, the intertwining of $\Delta_{Z}=\Delta_{Z}^{\prime}$ is obvious and the intertwining of $\Delta_{X}=\Delta_{X}^{\prime}$ follows from the decomposition $\Delta_{X}=\partial_{|X|}^{2}+$ $(\operatorname{dim} \mathbf{v}-1)|X|^{-1} \partial_{|X|}+|X|^{-2} \Delta_{S}$.

Choose an orthonormal basis $\left\{e_{0}, e_{1}, \ldots, e_{l-1}\right\}$ on the Z-space such that $e_{0}=A$. The Greek characters are used for the indices $\{0,1, \ldots, l-1\}$ and the Latin characters are used for the indices $\{1, \ldots, l-1\}$. Then $D_{c} \bullet=D_{c}^{\prime} \bullet$, furthermore

$$
D_{0} \bullet \Theta_{Q}(X)=\mathbf{i} \Theta_{Q}(X) \quad, \quad D_{0} \bullet \bar{\Theta}_{Q}(X)=-\mathbf{i} \bar{\Theta}_{Q}(X)
$$

and

$$
D_{c} \bullet \Theta_{Q}=\left\langle\mathbf{Q}, \mathbf{J}_{c}^{(a, b)}(X)\right\rangle=-\bar{\Theta}_{\mathbf{J}_{c}(Q)} \quad, \quad D_{c} \bullet \bar{\Theta}_{Q}=-\Theta_{\mathbf{J}_{c}(Q)}
$$

(Let us mention that the switching of the conjugation in (4.12) is due to the equation $\left.\mathbf{J}_{0} \mathbf{J}_{c}=-\mathbf{J}_{c} \mathbf{J}_{0}\right)$. Therefore $\kappa^{*} D_{\alpha} \bullet\left(\psi^{*}\right)=D_{\alpha}^{\prime} \bullet \kappa^{*}\left(\psi^{*}\right)$ for any $\psi^{*} \in \mathbf{P}_{S}^{(r)}$. Since $\kappa=T^{\prime} \circ \kappa^{*} \circ T^{-1}$, we also have $\kappa D_{\alpha} \bullet(\psi)=D_{\alpha}^{\prime} \bullet \kappa(\psi)$ and thus the terms $\sum \partial_{\alpha} D_{\alpha} \bullet$ and $\sum \partial_{\alpha} D_{\alpha}^{\prime} \bullet$ in (1.6) are intertwined by the $\operatorname{map} \kappa$.

Only the term $(1 / 4) \sum\left\langle\mathbf{J}_{\alpha}(X), \mathbf{J}_{\beta}(X)\right\rangle \partial_{\alpha \beta}$ should be considered yet.

First notice that on the Heisenberg-type groups, particularly on $\mathbf{H}_{3}^{(a, b)}$, this operator is nothing but (1/4) $|X|^{2} \Delta_{Z}$. Therefore the Intertwining Property of $\kappa$ is perfectly established in these special groups and (later we see that) also on their solvable extensions. Particularly, the Intertwining Property is established for the Striking Examples and only the intertwining of the boundary conditions should be scrutinized to proving the Isospectrality Theorem.

We turn back to the investigation of the considered operator on general spaces. Since $\mathbf{J}_{0} \circ \mathbf{J}_{c}$ is a skew symmetric endomorphism, $\left\langle\mathbf{J}_{0}(X), \mathbf{J}_{c}(X)\right\rangle=$ 0 . Therefore this operator is the same on the $\sigma_{\mathbf{A}}$-equivalent spaces $N$ and 
$N^{\prime}$. Yet, for the sake of completeness we should prove that the $\kappa$ maps a function of the form

$$
\left\langle\mathbf{J}_{c}^{(a+b, 0)}(X), \mathbf{J}_{d}^{(a+b, 0)}(X)\right\rangle h_{(r)}\left(\Theta^{p} \bar{\Theta}^{r-p}\right):=\mathbf{J}_{c d}(X) h_{(r)}\left(\Theta^{p} \bar{\Theta}^{r-p}\right)
$$

to a function of the very same form on $N^{\prime}$.

This problem can be simplified by the Spherical Decomposition Theorem (4.7). By this Theorem, the radial spherical harmonics span all the eigensubspaces $\mathbf{H}^{(r)}$, therefore, it is enough to consider the real functions of the form $K_{(r)}(X)=J_{c d}(X) h_{/(r)}\langle Q, X\rangle^{r}$. In this case the function $h_{/(r)}\langle Q, X\rangle^{r}$ is nothing but a constant multiple of the function $H_{(r)}(Q, X)$.

To figure out $\kappa\left(K_{(r)}\right)$, first the spherical decomposition of $K_{(r)}$ should be computed. By the "Technique" establishing the coefficients $A_{s_{i}}$ in (4.9), the component spherical harmonics are built up by the functions of the form

$$
\begin{gathered}
\langle Q, X\rangle^{p}, J_{c d}(X)\langle Q, X\rangle^{q} \\
\left\langle\left(J_{c} J_{d}+J_{d} J_{c}\right)(Q), X\right\rangle^{s}\langle Q, X\rangle^{v}=\left\langle Q_{c d}, X\right\rangle^{s}\langle Q, X\rangle^{v}
\end{gathered}
$$

such that the combinational coefficients depends only on the constants $r, p, s, v, \operatorname{Tr} J_{c} \circ J_{d}$ and on $\left\langle\mathbf{J}_{c}(Q), \mathbf{J}_{d}(Q)\right\rangle$.

The function $\mathbf{J}_{c d}(X)$ should be further scrutinized. It can be written in the form

$$
\begin{gathered}
\mathbf{J}_{c d}(X)=\sum_{i=1}^{K} \frac{1}{4}\left\{\left(\left\langle X, \mathbf{Q}_{c i}\right\rangle\left\langle X, \overline{\mathbf{Q}}_{d i}\right\rangle+\left\langle X, \overline{\mathbf{Q}}_{c i}\right\rangle\left\langle X, \mathbf{Q}_{d i}\right\rangle\right)+\right. \\
\left.\left(\left\langle X, \mathbf{Q}_{c i}\right\rangle\left\langle X, \mathbf{Q}_{d i}\right\rangle+\left\langle X, \overline{\mathbf{Q}}_{c i}\right\rangle\left\langle X, \overline{\mathbf{Q}}_{d i}\right\rangle\right)\right\}=\mathbf{J}_{c d}^{(1)}(X)+\mathbf{J}_{c d}^{(2)}(X),
\end{gathered}
$$

where $E_{1}, \ldots, E_{K}$ is an orthonormal basis on the X-space $(K=k(a+b))$ and $\mathbf{Q}_{e i}=\mathbf{J}_{e}^{(a, b)}\left(\mathbf{E}_{i}\right)$. The proof of this formula immediately follows from

$$
\mathbf{J}_{c d}(X)=\sum_{i}\left\langle\mathbf{J}_{c}(X), E_{i}\right\rangle\left\langle\mathbf{J}_{d}(X), E_{i}\right\rangle \quad, \quad E_{i}=\frac{1}{2}\left(\mathbf{E}_{i}+\overline{\mathbf{E}}_{i}\right) .
$$

Notice that the second function in (4.15) is a spherical harmonic function on the unit sphere $S$, having a vanishing integral on $S$. This statement follows from formulas $\left\langle\mathbf{Q}_{1}, \mathbf{Q}_{2}\right\rangle=\left\langle\overline{\mathbf{Q}}_{1}, \overline{\mathbf{Q}}_{2}\right\rangle=0$. By the very same reason, also the other two integrals vanish in the spherical expansion of this function. I. 
e., the function $\mathbf{J}_{c d}^{(2)}(X)$ vanishes and the simplification $\mathbf{J}_{c d}(X)=\mathbf{J}_{c d}^{(1)}(X)$ can be introduced in formula (4.15).

By the substitution $Q=(1 / 2)(\mathbf{Q}+\overline{\mathbf{Q}})$ we get that the componentspherical-harmonics in the decomposition of the function $K_{(r)}$ are the linear combinations of the functions of the form

$$
\Theta_{Q}^{p-s} \bar{\Theta}_{Q}^{r-p-s}, \mathbf{J}_{c d} \Theta_{Q}^{p-s} \bar{\Theta}_{Q}^{r-p-s},\left(\Theta_{Q_{c d}}+\bar{\Theta}_{Q_{c d}}\right) \Theta_{Q}^{p-v} \bar{\Theta}_{Q}^{r-p-v}
$$

such that the combinational coefficients depends only on the constants $r, p, s, v, \operatorname{Tr} J_{c} \circ J_{d}$ and on $\left\langle\mathbf{J}_{c}(Q), \mathbf{J}_{d}(Q)\right\rangle$.

The considered problem can be settled by representing $J_{c d}$ in the form (4.15) in formula (4.17). In fact, the coefficients discussed above are the same on both spaces (since they do not depend on the unit anticommutator $\left.\mathbf{J}_{0}^{(a, b)}\right)$. By the Technique establishing the coefficients $A_{s_{i}}$ in formula (4.9) we get that the pre-images (with respect to the map $T$ ) of the considered component-spherical-harmonics are combinations of appropriate functions (gotten from (4.17)) such that the coefficients do not depend on $\mathbf{J}_{0}^{(a, b)}$. This completely proves that the $\kappa$ maps a function $K_{(r)}$ (or, even a more complicated function, described (4.13)) to an appropriate function desired in this problem.

To complete the proof of the Isospectrality Theorem, we have to prove yet that the intertwining map $\kappa$ does not change the boundary conditions.

Since the functions of the form $\varphi(|X|, Z)$ are mapped to the very same function by the $\kappa$, furthermore, in any of the considered cases the Dirichlet boundary condition imposes restrictions only on these functions, the $\kappa$ obviously preserves the Dirichlet boundary conditions. Also the Neumann boundary condition is preserved.

In fact, the coefficient functions in (3.2) are the same on $\sigma$-equivalent spaces. Furthermore, by (4.11) and (4.12), the $\kappa$ intertwines the directional derivatives with respect to the radial vectors $X$ as well as with respect to the vectors $\mathbf{J}_{\alpha}(X)$. Therefore $\nu(\phi)=0$ if and only $\nu^{\prime} \phi^{\prime}=0$.

By summing up, we have

Main Theorem 4.3. The operator $\kappa=T^{\prime} \circ \kappa^{*} \circ T^{-1}$ intertwines the corresponding Laplacians and boundary conditions. Therefore the metrics on the considered $\sigma_{\mathbf{A}}$-equivalent spaces $N_{\mathbf{z}}^{(a, b)}$ and $N_{\mathbf{z}}^{\left(a^{\prime}, b^{\prime}\right)}$ with $a+b=a^{\prime}+b^{\prime}$ are both Dirichlet and Neumann isospectral on any of the regions described in the Ball- or Ball $\times$ Torus-cases.

According to Theorem 2.1, these constructions provide a wide range of isospectral spaces with different local geometries. Among these examples 
we find the H-type group $\mathbf{H}_{3}^{(a, b)}$. In this case the endomorphism algebra $J_{\mathbf{R}^{3}}$ forms a Lie algebra. All the endomorphism spaces containing this Lie algebra such that, say $\mathbf{J}_{1}$ is an anticommutator in the whole space (the wide range of these possibilities are described at the beginning of this paragraph!) also provide such examples.

It should be mentioned that these constructions provide the first examples for pairs of locally non-isometric yet Dirichlet and Neumann isospectral manifolds constructed on the most simple simply connected domains diffeomorphic to euclidean balls.

Remark 4.4 All the above statements can be easily extended to the more general cases, when $\mathbf{J}_{A}$ is just a non-degenerated anticommutator. In this case, first, introduce the unit anticommutator $A_{0}$ by an appropriate rescaling of $A$. This $A_{0}$ may not be in the endomorphism space, however, it is commuting with $A$ and it is anticommuting with the elements of $\mathbf{J}_{A^{\perp}}$. Then establish $\kappa$ by using $A_{0}$. Notice that the function $\mathbf{J}_{00}(X)$ is not equal to the function $|X|^{2}$ in general. However, formulas (4.14) and (4.15) remain true in the same form with respect to this function, which proves the statement completely.

Actually the Theorem can be stated in a more general form:

Main Theorem 4.3'. Let $\mathbf{J}_{\mathbf{z}}=\mathbf{A} \oplus \mathbf{A}^{\perp}$ and $\mathbf{J}_{\mathbf{z}}^{\prime}=\mathbf{A}^{\prime} \oplus \mathbf{A}^{\prime \perp}$ be endomorphism spaces with anticommutators such that the endomorphisms are acting on the same space $\mathbf{v}$ furthermore $\mathbf{A}^{\perp}=\mathbf{A}^{\perp}$ and the anticommutators $A$ and $A^{\prime}$ are isospectral (conjugate). Then the Ball-type domains are both Dirichlet and Neumann isospectral with respect to the metrics $g$ and $g^{\prime}$ of the spaces $N_{J}$ and $N_{J^{\prime}}$.

This more general Theorem is proven in [Sz4]. By this more general Theorem, one can construct isospectral spaces by deforming the anticommutator $A$ isospectrally, while keeping the orthogonal complement $\mathbf{A}^{\perp}$ unchanged. The full exploration of this idea is given in [Sz4].

This exploration includes the complete description of the $E S W_{A}$ 's in a Matrix Representation Theorem as well as the complete description of all possible non-trivial isospectral deformations of an anticommutator. It turns out that the continuous deformations are always trivial, providing isometric spaces. However, beside the $\sigma_{\mathbf{A}}$-deformations we point out a cornucopia of deformations providing spectrally inequivalent Endomorphism Spaces. They represent a completely new type of examples ever constructed in this field. For instance, these metrics are isospectral only on the Ball- and Sphere-type domains and they are not isospectral on the Ball $\times$ Torus-type 
domains or on their boundaries.

Remark 4.5 Using (3.12) and (3.13), the above Isospectrality Theorem can be trivially generalized to the solvable spaces $S N_{\mathbf{z}}^{(a, b)}$ and the Theorem remains true in the very same form.

In both cases the Theorem can be established also on the boundaries of these domains. Extremely interesting examples are provided by the geodesic spheres with the same radius on the harmonic spaces $S \mathbf{H}_{3}^{(a, b)}$, where $a+b$ is the same dimension number. It turns out that these spheres are isospectral and one of them is homogeneous while the others are inhomogeneous. We consider these Examples in $\S 6$.

\section{5. $\oint$ Spaces with isotonal curvatures. Some results on invariant forms}

We study the analogous isospectrality properties of the Laplace- and the non-relativistic Dirac-spectra on forms in a separate paper. Here we describe some results only about the spectra of the Laplacian acting on invariant differential forms. In the end we consider also the spectrum of the curvature operator acting as a symmetric endomorphism on the 2-forms. We prove that $\sigma$-equivalent spaces have isotonal curvature. Both type of these spectra have an important impact on the corresponding spectra of the general forms.

We start by describing the action of $d$ and $\delta$ operators on invariant differential forms.

The invariant differential forms can be expressed by the 1-forms $\theta_{i}$ and $\vartheta_{\alpha}$ introduced in (3.16), since all these differential forms can be expressed as a linear combination of the forms:

$$
\theta_{\left\{i_{1}, \ldots, i_{p_{1}}, \alpha_{1}, \ldots, \alpha_{q}\right\}}=\theta_{i_{1}} \wedge \cdots \wedge \theta_{i_{p_{1}}} \wedge \vartheta_{\alpha_{1}} \wedge \cdots \wedge \vartheta_{\alpha_{q}},
$$

where $i_{1}<\cdots<i_{p_{1}}$ and $\alpha_{1}<\cdots<\alpha_{q}$.

The formulas (3.17) completely describe the action of the d-operator on invariant differential forms. Therefore we should deal only with the $\delta$ 
operator. From (1.16) we straightforwardly get

$$
\begin{gathered}
\delta \theta_{i}=0 \quad, \quad \delta \vartheta_{\alpha}=0 \quad, \quad \delta\left(\theta_{i} \wedge \vartheta_{\alpha}\right)=0 \\
T_{i j}=\delta\left(\theta_{i} \wedge \theta_{j}\right)=-\vartheta_{\left[E_{i}, E_{i}\right]}=-\sum_{\alpha=0}^{l}\left\langle\mathbf{J}_{\alpha}\left(E_{i}\right) . E_{j}\right\rangle \vartheta_{\alpha}, \\
\delta\left(\theta_{i_{1}} \wedge \cdots \wedge \theta_{i_{p}} \wedge \vartheta_{\alpha_{1}} \wedge \cdots \wedge \vartheta_{\alpha_{q}}\right)= \\
\sum_{r\langle s,} C \cdots \wedge \theta_{i_{r-1}} \wedge \theta_{i_{r+1}} \wedge \cdots \wedge \theta_{i_{s-1}} \wedge \theta_{i_{s+1}} \wedge \cdots \wedge \vartheta_{\alpha_{q}} \wedge T_{i_{r} i_{s}},
\end{gathered}
$$

where $\mathrm{C}$ is a combinatorial constant depending on $p$ and $q$.

The Laplacian acting on invariant differential forms can be expressed by these formulas.

In the following we describe some results about the spectra of the Laplacian acting on invariant differential forms. Such a description of the spectra on the invariant forms is an important step also to the description of the spectra on the general forms. In fact, the general forms are spanned by the forms $F \theta_{\left\{i_{1}, \ldots, \alpha_{q}\right\}}$, and the action of the Laplacian on such a form is

$$
\Delta F \theta_{\left(i_{1}, \ldots, \alpha_{q}\right)}=(\Delta F) \theta+2 \nabla_{\operatorname{grad} F} \Theta+F \Delta \theta .
$$

Once the spectrum problem is solved on functions and on the invariant differential forms (i. e. on the ends of the above formula), the general problem requires the investigation of the middle operator $\nabla_{\operatorname{gradF}} \theta$.

In this paper we determine the spectrum of the Laplacian only on invariant 1- and 2-forms.

Since the $\delta$-operator is trivially acting on 1 -forms, $\Delta=-\delta d$ in this case. By (3.17) and (5.2) we get

Proposition 5.1. The eigenvectors of the Laplacian acting on the invariant 1-forms are divided into two classes.

(A) The $X$-forms $\theta_{Q}$ with eigenvalue 0 .

(B) The $Z$-forms $\vartheta_{Z_{\alpha}}$ such that $Z_{\alpha}$ is an eigenvector of the quadratic form $T\left(Z, Z^{*}\right)=\operatorname{Tr} \mathbf{J}_{Z} \circ \mathbf{J}_{Z^{*}}$. The eigenvalues are the negatives of the eigenvalues of this quadratic form.

Therefore, the Laplacians acting on invariant 1-forms are isospectral on $\sigma$-equivalent spaces.

It should be mentioned that this spectrum is the same also on the members of the continuous family of two step nilpotent Lie groups constructed 
by Gordon and Wilson in [GW3] (see Corollary 4.2 of this article). In view of Schueth's Theorem (namely, these spaces are not isospetral on general 1-forms), the isospetrality on functions and on invariant 1-forms does not imply the isospectrality on the general 1-forms. (Though Schueth's Theorem concerns the boundary of these manifolds, it is extremely probable that the statement is valid on the manifolds as well.

Next we determine the spectrum of the Laplacian acting on the invariant 2 -forms.

For the sake of simplicity we suppose that the inner product on the Zspace is the natural inner product $T\left(Z_{1}, Z_{2}\right)=\operatorname{Tr} \mathbf{J}_{Z_{1}} \circ \mathbf{J}_{Z_{2}}$. If we use a different inner product, then, like in the previous statement, the eigenvalues of the quadratic form $\mathrm{T}$ emerge in the following spectrum.

The space $\wedge^{2}$ of the invariant 2 -forms splits into the following 3 invariant subspaces:

$$
\theta_{\mathbf{v}} \wedge \theta_{\mathbf{v}} \quad, \quad \vartheta_{\mathbf{z}} \wedge \vartheta_{\mathbf{z}} \quad, \quad \theta_{\mathbf{v}} \wedge \vartheta_{\mathbf{z}}
$$

By (3.23) and (5.2), the Laplacian is acting on these invariant subspaces as follows.

$$
\begin{gathered}
\Delta \theta_{i} \wedge \theta_{j}=-\sum_{\alpha} \mathbf{J}_{\alpha i j} \mathbf{J}_{\alpha}^{p q} \theta_{p} \wedge \theta_{q} \quad, \quad \Delta \vartheta_{\alpha} \wedge \vartheta_{\beta}=-2 \vartheta_{\alpha} \wedge \vartheta_{\beta} \\
\Delta \theta_{i} \wedge \vartheta_{\alpha}=\theta_{i} \wedge \vartheta_{\alpha}+\sum_{\beta, j}\left\langle\mathbf{J}_{\beta}\left(E_{i}\right), \mathbf{J}_{\alpha}\left(E_{j}\right)\right\rangle \theta_{j} \wedge \vartheta_{\beta}= \\
\theta_{i} \wedge \vartheta_{\alpha}+\sum_{\beta, j}\left(\left\langle\mathbf{J}_{\alpha}\left(E_{i}\right), \mathbf{J}_{\beta}\left(E_{j}\right)\right\rangle+\left\langle\left[\mathbf{J}_{\alpha}, \mathbf{J}_{\beta}\right]\left(E_{i}\right), E_{j}\right\rangle\right) \theta_{j} \wedge \vartheta_{\beta}
\end{gathered}
$$

One can interpret these formulas as follows.

(A) In case of the invariant subspace $\theta_{\mathbf{v}} \wedge \theta_{\mathbf{v}}$, a 2 -form $u^{i j} \theta_{i} \wedge \theta_{j}$ can be considered as a skew endomorphism. Then the Laplacian is the -1 times of the projection onto the subspace $\mathbf{J}_{\mathbf{z}}$ in this case. Therefore the Laplacian has the eigenvalue -1 with multiplicities $l$ and the eigenvalue 0 with multiplicities equal to the codimension of $\mathbf{J}_{\mathbf{z}}$ in the whole space of skew endomorphisms.

The second case should not be interpreted.

(B) With respect to the invariant space $\theta_{\mathbf{v}} \wedge \vartheta_{\mathbf{z}}$ notice that the restricted Laplacian onto this space is the same on $\sigma$-equivalent spaces. Therefore the $\sigma$-equivalent spaces are isospectral on the invariant 2 -forms. 
Proposition 5.2. The Laplacians acting on the invariant 2-forms are isospectral on $\sigma$-equivalent spaces.

Finally we consider the curvature operators on these spaces. By (1.9) we obviously get

Proposition 5.3. Two $\sigma$-equivalent spaces have identical Ricci curvature at the origin.

Now we consider the spectrum of the curvature operator $R_{i j}{ }^{p q}$ acting as a symmetric endomorphism on the space of 2 -forms. This spectrum is called the $\wedge^{2}-$ spectrum of the curvature operator. By (1.18), this spectrum has a great impact on the Laplacian spectra on forms. Yet, surprisingly enough, this spectrum distinguishes the $\sigma$-equivalent spaces, since we have

Proposition 5.4. The curvatures of the $\sigma$-equivalent spaces $N_{\mathbf{z}}^{(a, b)}$ and $N_{\mathbf{z}}^{\left(a^{\prime}, b^{\prime}\right)}$ with $a b a^{\prime} b^{\prime} \neq 0$ are always isotonal (i. e., the elements of the spectra are the same but the multiplicities may be different).

In many cases they are strictly isotonal (not isospectral) unless $(a, b)=$ $\left(a^{\prime}, b^{\prime}\right)$ up to an order. For instance, this statement is true on the spaces $\mathbf{H}_{3}^{(a, b)}$.

A general criteria is formulated in the following proof for this Isotonality Statement: If $0 \neq a b \neq a^{\prime} b^{\prime} \neq 0$, the curvatures are strictly isotonal iff the spectrum on the mixed boxes changes when it is multiplied by -1 .

The curvature of the space $N_{\mathbf{z}}^{(a+b, 0)}$ is subtonal to the curvature of the above spaces, $i$. e. the corresponding tonal spectrum on $N_{\mathbf{z}}^{(a+b, 0)}$ is a subset of the common tonal spectrum of the other spaces.

Proof. Also in this case we decompose the space $\wedge^{2}$ of the 2 -forms into invariant subspaces. Unlike in case of the Laplace spectrum on the invariant 2-forms, now the decomposition $\mathbf{v}=\mathbf{v}^{(a)} \oplus \mathbf{v}^{(b)}$, where $\mathbf{v}^{(a)}=\mathbf{R}^{n a} ; \mathbf{v}^{(b)}=$ $\mathbf{R}^{n b}$ (see (2.2)), plays an important role. First we decompose the space $\wedge^{2}$ into the following 3 invariant subspaces:

$$
\begin{gathered}
\mathbf{D}=\theta_{\mathbf{v}^{(a)}} \wedge \theta_{\mathbf{v}^{(a)}} \oplus \theta_{\mathbf{v}^{(b)}} \wedge \theta_{\mathbf{v}^{(b)}} \oplus \vartheta_{\mathbf{z}} \wedge \vartheta_{\mathbf{z}} \\
\mathbf{F}=\theta_{\mathbf{v}^{(a)}} \wedge \theta_{\mathbf{v}^{(b)}} \quad, \quad \mathbf{G}=\theta_{\mathbf{v}} \wedge \vartheta_{\mathbf{z}}
\end{gathered}
$$

On the space $\mathbf{G}$, the action is described by

$$
\theta_{i} \wedge \vartheta_{\alpha} \rightarrow \sum_{j, \beta}-\frac{1}{4}\left\langle\mathbf{J}_{\beta}\left(E_{i}\right), \mathbf{J}_{\alpha}\left(E_{j}\right)\right\rangle \theta_{j} \wedge \vartheta_{\beta}
$$


In Proposition 5.2 we proved that this operator is isospectral on any pair of $\sigma$-equivalent spaces.

On the space $\mathbf{D}$, the curvature is acting by the components described by the first and the last lines in (1.8). In this case we need a more refined decomposition into invariant subspaces.

In the following $\mathbf{v}_{r}^{(a)} \simeq \mathbf{R}^{n}$ means the $r^{t h}$ component-space of $\mathbf{v}^{(a)}$. The component-space $\mathbf{v}_{s}^{(b)}$ is defined similarly.

The forms forming the subspace

$$
\mathbf{D g}=\sum_{r} \theta_{\mathbf{v}_{r}^{(a)}} \wedge \theta_{\mathbf{v}_{r}^{(a)}} \oplus \sum_{s} \theta_{\mathbf{v}_{s}^{(b)}} \wedge \theta_{\mathbf{v}_{s}^{(b)}} \oplus \vartheta_{\mathbf{z}} \wedge \vartheta_{\mathbf{z}}
$$

are said to be diagonal forms.

The space $\mathbf{D g}^{\perp} \subset \mathbf{D}$ of the orthogonal forms in $\mathbf{D}$ is spanned by the blocks $\mathbf{v}_{r}^{(a)} \wedge \mathbf{v}_{r^{*}}^{(a)}$ and $\mathbf{v}_{s}^{(b)} \wedge \mathbf{v}_{s^{*}}^{(b)}$, where $s \neq s^{*}$ and $r \neq r^{*}$. Note that on these blocks only the component

$$
\begin{aligned}
& u^{i_{r} j_{r^{*}}} \theta_{i_{r}} \wedge \theta_{j_{r^{*}}} \rightarrow \frac{1}{2} \sum_{\alpha} u^{i_{r} j_{r^{*}}} \mathbf{J}_{\alpha i_{r}}{ }^{p_{r}} \mathbf{J}_{\alpha i_{r}^{*}} p_{r^{*}}^{*} \theta_{p_{r}} \wedge \theta_{p_{r^{*}}^{*}}, \\
& u^{i_{s} j_{s^{*}}} \theta_{i_{s}} \wedge \theta_{j_{s^{*}}} \rightarrow \frac{1}{2} \sum_{\alpha} u^{i_{s} j_{s^{*}}} \mathbf{J}_{\alpha i_{s}}{ }^{q_{s}} \mathbf{J}_{\alpha i_{s}^{q_{s}^{*}}} \theta_{q_{s}} \wedge \theta_{q_{s^{*}}^{*}}
\end{aligned}
$$

of the curvature operator (which comes from the second and third term of the first line of (1.8)) acts non-trivially, leaving this space invariant. Furthermore this operator is the same on any of the considered spaces, therefore the curvature is isospectral on these invariant spaces.

We can prove the same isospectrality also on the invariant space $\mathbf{D g}$ of the diagonal forms.

In fact, on a space $N_{\mathbf{z}}^{(a, b)}$ consider the map $\tau: \mathbf{D g} \rightarrow \mathbf{D g}$ such that $\tau=i d$ on $\theta_{\mathbf{v}^{(a)}} \wedge \theta_{\mathbf{v}^{(a)}}$ and $\tau=-i d$ on $\theta_{\mathbf{v}^{(b)}} \wedge \theta_{\mathbf{v}^{(b)}}$. Then the $\tau$ intertwines the curvatures of the spaces $N_{\mathbf{z}}^{(a+b, 0)}$ and $N_{\mathbf{z}}^{(a, b)}$ on this invariant space $\mathbf{D g}$.

None of the spectrums investigated so far distinguishes the considered spaces. These spaces are distinguishable by the curvature spectrum on the invariant space $\mathbf{F}$. This space splits into the direct sum of the "mixed" boxes $\theta_{\mathbf{v}_{r}^{(a)} \wedge \mathbf{v}_{s}^{(b)}}$. Each of these boxes is invariant on which only the component

$$
u^{i_{r} j_{s}} \theta_{i_{r}} \wedge \theta_{j_{s}} \rightarrow \frac{1}{2} \sum_{\alpha} u^{i_{r} j_{s}} \mathbf{J}_{\alpha i_{r}}{ }^{p_{r}} \mathbf{J}_{\alpha i_{s}}{ }^{q_{s}} \theta_{p_{r}} \wedge \theta_{q_{s}}
$$


of the curvature is acting non-trivially. On a pair of such boxes, the spectrum is the same. More precisely, this spectrum is the negative of the spectrum what we get on the boxes of the invariant space $\mathbf{D g}^{\perp}$.

If this spectrum does change when it is multiplied by -1 , the curvatures on the spaces $N_{\mathbf{z}}^{(a+b, 0)}$ and $N_{\mathbf{z}}^{(a, b)}$ can not be isospectral. However, the first is subtonal to the second one by the above arguments. If $0 \neq a b \neq a^{\prime} b^{\prime} \neq 0$, the spaces $N$ and $N^{\prime}$ have different number of mixed boxes. Therefore the curvature spectra of these spaces on the space of mixed boxes are just isotonal.

Let us mentioned that the above mentioned spectrum on a mixed box of the space $\mathbf{H}_{3}^{(a, b)}$ is $\left\{\lambda=-1 / 2, m_{\lambda}=12 ; \lambda=3 / 2, m_{\lambda}=4\right\}$, where $m_{\lambda}$ means the multiplicity of the corresponding eigenvalue. This spectrum changes if we multiply it by -1 , therefore these $\sigma$-equivalent spaces have strictly isotonal curvatures (unless $(a, b)=\left(a^{\prime}, b^{\prime}\right)$ up to an order).

\section{6. $\oint$ Constructing the Striking Examples}

The proofs of the Isospectrality Theorems on the boundary manifolds are incorporated into [Sz4]. We have to point out that the proof of the isospectrality itself is the easy part of these considerations, since from the explicit expression of the Laplacian on $\partial D$ one can get immediately that an appropriate Intertwining Operator can be defined in the very same way as on the ambient space. Only the functions $\varphi(|X|, Z)$ (resp. $\varphi(|X|, Z, t)$ in the solvable case) should be changed to functions of the form $\varphi(\delta(Z), Z)=$ $\phi(Z)$ (resp. of the form $\varphi(\delta(Z, t), Z, t)=\phi(Z, t)$ ), where $Z$ varies on the boundary. However, the Non-Isometry Proofs are much more complicated on the boundaries than on the ambient spaces, because the boundaries are inhomogeneous spaces and we lose the technique of the homogeneous spaces.

In the following we describe more details on the special H-type spaces $\mathbf{H}_{3}^{(a, b)}$ and on their solvable extensions $S \mathbf{H}_{3}^{(a, b)}$, to discover the most striking examples of our constructions. Namely, the metrics on the geodesic spheres with the same radius are isospectral on the spaces $S \mathbf{H}_{3}^{(a, b)}$ with the same $a+b$, despite the radical differences shown by the isometry groups: The isometry groups have different dimensions on these spaces and, even more surprisingly, the geodesic spheres are homogeneous on the 2-point homogeneous space $S \mathbf{H}_{3}^{(a+b, 0)}$, while the metrics on the other spheres are locally inhomogeneous.

In the following we consider domains, $D$, such that the boundary $\partial D$ 
is described by a function of the form $|X|=\delta(|Z|)$ in the nilpotent case and of the form $|X|=\delta(|Z|, t)$ in the solvable case. I. e., both the X- and the Z-cross sections with this boundary are spheres denoted by $S_{X}(Z)$ and $S_{Z}(X)$ in the nilpotent case and by $S_{X}(Z, t)$ and $S_{Z}(X, t)$ in the solvable case. Let us mention that the geodesic spheres around the origin are such hypersurfaces on these spaces. More precisely, the geodesic spheres with the same radius $R$ around the origin are the same point-sets on these groups and they are described by the same equation which has a relatively complicated implicit form, $\sigma(R,|X|,|Z|)=0$, in the nilpotent case (see [CDKR] and [Sz2] for more details) and it has the more simple explicit form

$$
|X|^{2}=4\left(\left(e^{R}+e^{-R}+2\right) t-|Z|^{2}\right)^{\frac{1}{2}}-4(t+1)
$$

in the solvable case [Sz4].

The Laplacian on the boundary $\partial D$ can be computed by standard arguments. The corresponding formulas are established in [Sz4]. One can check the idea of these computations also in [Sz3] (cf. formulas (3.1)-(3.3)). In the nilpotent case we get

$$
\tilde{\Delta}=\Delta_{S_{X}(Z)}+\left(1+\frac{1}{4} \delta^{2}(|Z|)\right) \Delta_{S_{Z}(X)}+\sum_{\alpha=1}^{3}\left(\partial_{\alpha}-Z_{u \alpha}\right) D_{\alpha} \bullet
$$

The last term is nothing but the restriction of the operator $\sum_{\alpha} \partial_{\alpha} \mathbf{J}_{\alpha} \bullet$ onto the boundary $\partial D$.

In the solvable case we have

$$
\begin{gathered}
\tilde{\Delta}=t \Delta_{S_{X}}+\left(t^{\frac{1}{2}}+\frac{1}{4} t|X|^{2}\right) \Delta_{S_{Z}}+ \\
t \sum_{\alpha=1}^{3}\left(\partial_{\alpha}-Z_{u \alpha}\right) D_{\alpha} \bullet+c^{2} t^{2}\left(\partial_{t}-\boldsymbol{\mu}_{t}\right)^{2}-c^{2}\left(2+\frac{k}{2}\right) t\left(\partial_{t}-\boldsymbol{\mu}_{t}\right),
\end{gathered}
$$

where $\boldsymbol{\mu}_{t}=C(|Z|, t) \partial_{t}$ is introduced in (3.13).

Restrict the functions (4.9) onto the boundary $\partial D$ and define the map $\tilde{\kappa}$ on these restricted functions again by the formula (4.10). The same idea is applicable to the solvable extensions. Then by the same proof established on the ambient spaces we get that this map intertwines the Laplacians on the considered function spaces. Therefore we have 
Theorem 6.1. If $a+b=a^{\prime}+b^{\prime}$, the metrics induced on the Sphere-type boundary $\partial D$ are isospectral on the spaces $\mathbf{H}_{3}^{(a, b)}$ and $\mathbf{H}_{3}^{\left(a^{\prime}, b^{\prime}\right)}$ as well as on the solvable extensions $S \mathbf{H}_{3}^{(a, b)}$ and $S \mathbf{H}_{3}^{\left(a^{\prime}, b^{\prime}\right)}$.

Particularly we get that the metrics on the geodesic spheres with the same radius of these spaces are isospectral.

The Non-Isometry Proofs can be established by a simple modification of the Proofs given in [Sz3] on the SpherexTorus-type manifolds. Actually, three different proofs are given there and each of them can be reconstructed also for the case of Sphere-type boundaries.

The details are as follows

At a point $(X, Z) \in \partial D$, let $\widetilde{z}$ be the tangent space of the sphere $S_{Z}(X)$, furthermore consider also the distribution $\rho^{(a, b)}=\mathbf{J}_{\mathbf{z}}^{(a, b)}(X) \subset T\left(S_{X}(Z)\right)$ on the sphere $S_{X}(Z)$ of the X-space. In the solvable case we consider also the 1-dimensional distribution $\widetilde{T}$ spanned by the unit tangent vector $\mathbf{t}$ of the t-parameter curves on the surface. Let $K^{(a, b)}$ be the distribution orthogonal to $\rho^{(a, b)} \oplus \widetilde{z}$ in the nilpotent case and it should be perpendicular also to $\widetilde{T}$ in the solvable case.

By computing the Ricci curvature on $\partial D$ it turns out that the $K^{(a, b)}$ is an eigensubspace of the Ricci operator and the eigenvalues, taken on this subspace, are completely different from the other eigenvalues. This statement concerns the nilpotent case as well as the solvable case.

For proving this Statement, the matrix of the Ricci operator is computed in a particularly chosen basis in [Sz4]. At a point $(X, Z)$ (resp. at $(X, Z, t))$ on the surface the orthonormal basis $\{\mathbf{i}, \mathbf{j}, \mathbf{k}\}$ on the Z-space is chosen such that $\mathbf{i}=Z_{u}$. Then $\mathbf{j}$ and $\mathbf{k}$ are tangent to the surface. If $\boldsymbol{\mu}_{X}$ (considered as a Lie algebra element) is the $\mathrm{X}$-component of the normal vector and $\boldsymbol{\mu}_{X u}$ denotes the normalized vector, then introduce the vectors (considered as Lie algebra elements)

$$
\widetilde{E}_{\mathbf{i}}=\mathbf{J}_{\mathbf{i}}\left(\boldsymbol{\mu}_{X u}\right), \widetilde{E}_{\mathbf{j}}=\mathbf{J}_{\mathbf{j}}\left(X_{u}\right), \widetilde{E}_{\mathbf{k}}=\mathbf{J}_{\mathbf{k}}\left(X_{u}\right) .
$$

Let us mentioned that the vectors $\widetilde{E}_{\mathbf{j}}$ and $\widetilde{E}_{\mathbf{k}}$ are tangent to the distribution $\rho^{(a, b)}$ while $\widetilde{E}_{\mathbf{i}}$ is not tangent to this distribution, expressing the fact that the subspaces $\rho^{(a, b)}$ and $\widetilde{z}$ are not perpendicular.

We consider an orthonormal basis $\left\{\widetilde{K}_{1}, \ldots, \widetilde{K}_{k-3}\right\}$ also on $K^{(a, b)}$ and the matrix of the Ricci operator $\widetilde{r}$ is computed with respect to the basis $\left\{\widetilde{K}_{1}, \ldots, \widetilde{K}_{k-3}, \widetilde{E}_{\mathbf{i}}, \widetilde{E}_{\mathbf{j}}, \widetilde{E}_{\mathbf{k}}, \mathbf{j}, \mathbf{k}\right\}$, in the nilpotent case. Then in [Sz4] we get 
that this matrix is of the form

$$
\widetilde{r}=\left(\begin{array}{cccc}
\epsilon I_{K} & 0 & 0 & 0 \\
0 & \left(\epsilon+E_{l l}\right) I_{l} & 0 & 0 \\
0 & 0 & \left(\epsilon+E_{L L}\right) I_{L} & E_{L \widetilde{z}} \\
0 & 0 & E_{\widetilde{z} L} & \left(\epsilon+E_{\widetilde{z z}}\right) I_{\widetilde{z}}
\end{array}\right),
$$

where $I_{K}, I_{\widetilde{z}}$ are unit matrices on the spaces $K, \widetilde{z}$ and $I_{L}$ (resp. $I_{l}$ ) is unit matrix on the space $L$ spanned by the vectors $\mathbf{j}$ and $\mathbf{k}$ (resp. on the 1-dimensional space $l$ spanned by $\mathbf{i}$ ). The entries $\epsilon, E_{l l}, E_{L L}, E_{\widetilde{z z}}$ are very complicated expressions depending on the function $\delta(|Z|)$ (cf. (4.20) in $[\mathrm{Sz} 4])$. Yet it turns out that the determinant

$$
\operatorname{det}\left(\begin{array}{cc}
E_{L L} I_{L} & E_{L \widetilde{z}} \\
E_{\widetilde{z} L} & E_{\widetilde{z z}} I_{\widetilde{z}}
\end{array}\right)
$$

is non-zero on an everywhere dense open set. This statement proves that on this set the Ricci tensor has distinct eigenvalues on the invariant subspaces $K$ and $\rho \oplus \widetilde{z}$.

In the solvable case the Ricci curvature $\widetilde{r}_{S}$ is computed with respect to the basis $\left\{\widetilde{K}_{1}, \ldots, \widetilde{K}_{k-3}, \widetilde{E}_{\mathbf{i}}, \widetilde{E}_{\mathbf{j}}, \widetilde{E}_{\mathbf{k}}, \mathbf{j}, \mathbf{k}, \mathbf{t}\right\}$. Then we get

$$
\widetilde{r}_{S}=\left(\begin{array}{ccccc}
\sigma I_{K} & 0 & 0 & 0 & 0 \\
0 & \left(\sigma+S_{l l}\right) & 0 & 0 & S_{l \mathbf{t}} \\
0 & 0 & \left(\sigma+S_{L L}\right) I_{L} & t^{\frac{1}{2}} S_{L \widetilde{z}} & 0 \\
0 & 0 & t^{\frac{1}{2}} S_{\widetilde{z} L} & \left(\sigma+S_{\widetilde{z z}}\right) I_{\widetilde{z}} & 0 \\
0 & S_{\mathbf{t} l} & 0 & 0 & \sigma+S_{\mathbf{t t}}
\end{array}\right)
$$

By the very same proof given in the nilpotent case we get that the eigenvalue $\sigma$ on the eigenspace $K$ is different from the other eigenvalues on an everywhere dense open set of a geodesic sphere and therefore this distribution is invariant by the actions of the isometries.

The First Non-Isometry Proof can be established as follows

For the vector fields $U$ and $V$ tangent to $\rho^{(a, b)} \oplus \widetilde{z}$ in the nilpotent case, resp. tangent to $\rho^{(a, b)} \oplus \widetilde{z} \oplus \widetilde{T}$ in the solvable case, let $L(U, V)$ be the orthogonal projection of $[U, V]$ onto $K^{(a, b)}$. The $L$ is obviously a tensor field of type $(2,1)$ on $\partial D$ and it is invariant with respect to the action of isometries on the space. By (2.4), this $L$ vanishes exactly at the points of the form $\left(X^{(a)}, Z\right)$ or $\left(X^{(b)}, Z\right)$, i. e. the induced metrics on the hypersurface $\partial D$ of the spaces $\mathbf{H}_{3}^{(a, b)}$ and $\mathbf{H}_{3}^{\left(a^{\prime}, b^{\prime}\right)}$ (resp. on their solvable extensions) can not be isometric unless $(a, b)=\left(a^{\prime}, b^{\prime}\right)$ up to an order. 
This Proof also proves that the isometries on the geodesic spheres of the spaces $S \mathbf{H}_{3}^{(a, b)}$ with $a b \neq 0$ are locally inhomogeneous and thus the Non-Isometry Proof is completely established for the Striking Examples.

In [Sz4] we describe two other proofs. In the second Non-Isometry Proof we trace back the problem to the ambient space by showing that the considered surfaces are isometric if and only if the ambient spaces are isometric.

First we describe a geometric interpretation of the distribution $K^{(a, b)}$. On the space $H_{3}^{(a, 0)}$ (resp. on $H_{3}^{(0, b)}$ ) the distribution $\rho^{(a, 0)}$ (resp. $\rho^{(0, b)}$ ) is integrable and the 3-dimensional integral manifolds are the fibers of a principal fiber bundle with the structure group $S O(3)$. This fibration is nothing but the quaternionic Hopf fibration and the factor space is the 2-point homogeneous quaternionic projective space [Be].

If $a>1$ (resp. $b>1$ ), the distribution $K^{(a, 0)}$ (resp. $\left.K^{(0, b)}\right)$ is an irreducible connection on this bundle with an irreducible curvature form $\omega(X, Y)=[X, Y]_{\rho} ; X, Y \in K$. This proves that $\left[K^{(a, b)}, K^{(a, b)}\right]_{\rho}=\rho^{(a, b)}$. Therefore the $K$ generates the whole tangent space on a sphere $S_{X}(Z)$ (resp. on $S_{X}(Z, t)$ ) by Lie brackets. Since the $K^{(a, b)}$ is invariant by the actions of isometries, the spheres $S_{X}$ must be mapped to such spheres by isometries. By (1.11) (resp. by the corresponding version on the solvable extension) we get that this action between these spheres is nothing but restriction of an orthogonal transformation, defined between the ambient $\mathrm{X}$-spaces. These orthogonal transformations define an extension of the considered isometry onto the ambient space, which turns out to be an isometry between the ambient spaces.

In the third Non-Isometry Proof we determine the isometries on the considered hypersurfaces. It turns out that in case of $a b \neq 0$, the group of isometries is the non-transitive group $O\left(\mathbf{H}^{a}\right) \times O\left(\mathbf{H}^{b}\right) \times S O(3)$ (semi direct product), in both of the nilpotent and the solvable cases, where $O\left(\mathbf{H}^{c}\right)$ is the quaternionic orthogonal group acting on $\mathbf{H}^{c}$. These groups are obviously different from the transitive isometry groups acting on the corresponding 2-point homogeneous space. By summing up we have

Theorem 6.2. The metrics on the same radius geodesic spheres are isospectral on a discrete family, $S \mathbf{H}_{3}^{(a, b)}$, of solvable $\sigma$-equivalent spaces. These spheres have different local geometries unless $(a, b)=\left(a^{\prime}, b^{\prime}\right)$ up to an order.

The geodesic spheres on a space $S \mathbf{H}_{3}^{(a+b, 0)}$ are homogeneous, while the spheres on the other spaces are locally inhomogeneous. This demonstrate the fact: "One can not hear the local homogeneity property even on the most simple closed manifolds, namely on spheres." 


\section{References}

[Be] A. Besse, Manifolds all of whose geodesics are closed, Springer-Verlag Berlin Heidelberg New York, 1978.

[BT] R. Brooks, R. Tse, Isospectral surfaces of small genus, Nagoya Math. J. 107 (1987), 13-24.

[Bu] P. Buser, Isospectral Riemann surfaces,, Ann. Inst. Fourier (Grenoble) 36 (1986), 167-192.

[CH] R. Courant, D. Hilbert, Methods of mathematical physics, Vol. I, Interscience, 1953.

[Cha] I. Chavel, Eigenvalues in Riemannian geometry, Academic Press, 1984.

[CDKR] M. Cowling, A. H. Dooley, A. Koranyi, F. Ricci, $H$-type groups and Iwasawa decompositions, Adv. in Math. 87 (1991), 1-41.

[CS] C. Croke, V. Sharafutdinov, Spectral rigidity of a compact negatively curved manifold, Topology 37 (1998), 1265-1273.

[D] E. DAMEK, Geometry of a semidirect extension of a Heisenberg type nilpotent group, Colloq. Math. 53 (1987), 255-268.

[DR] E. DAMEK, F. RICCI, A class of non-symmetric harmonic manifolds, Bull. Amer. Math. Soc. 27 (1992), 139-142.

[DG] D. DeTruck, C. Gordon, Isospectral deformations II., Com. Pure Appl. Math. 42 (1989), 1067-1095.

[E] P. Eberlein, Geometry of two-step nilpotent groups with a left invariant metric, Ann. Sci. École Norm. Sup. 27 (1994), 611-660.

[G1] C. Gordon, Isospectral closed Riemannian manifolds which are not locally isometric, J. Diff. Geom. 37 (1993), 639-649.

[G2] C. Gordon, Isospectral closed manifolds which are not locally isometric, Part II, Contemporary Mathematics: Geometry of the spectrum (R. Brooks, C. Gordon, P. Perry, eds.), vol. 173, AMS, 1994, 121-131.

[GGSW] C. Gordon, R. Gornet, D. Schueth, D. Webb, E. Wilson, Isospec tral deformations of closed Riemannian manifolds with different scalar curvature, Ann. Inst. Four., Grenoble 48 (1998), 593-607.

[GS] C. Gordon, Z.I. Szabó, Isospectral deformations on negatively curved manifolds with boundaries, Preprint.

[GW1] C. Gordon, E. N. Wilson, Isospectral deformations of compact solvmanifolds, J. Diff. Geom. 19 (1984), 241-256.

[GW2] C. Gordon, E. N. Wilson, The spectrum of the Laplacian on Riemannian Heisenberg manifolds, Michigan Math. J. 33 (1986), 253-271.

[GW3] C. Gordon, E. N. Wilson, Continuous families of isospectral Riemannian metrics which are not locally isometric, J. Diff. Geom. 47 (1997), 504-529.

[GWW] C. Gordon, D. Webb, S. Wolpert, One can not hear the shape of a drum, Bull. Amer. Math. Soc. 27, no. 1 (1992), 134-138.

[I] A. IKEDA, On lens spaces which are isospectral but not isometric, Ann. Sci. Ecole Norm. Sup. (4)13 (1980), 303-315. 
[K] A. Kaplan, Riemannian nilmanifolds attached to Clifford modules, Geom. Dedicata 11 (1981), 127-136.

[Ka] R. KARIDI, Ricci curvature and volume growth for leftinvariant Riemannian metrics on nilpotent and some solvable Lie groups, Geom. Dedicata 46 (1993), 249-277.

[M] J. Milnor, Eigenvalues of the Laplace operator on certain manifolds, Proc. Nat. Acad. Sci. U.S.A. (1964), 542.

[P] H. Pesce, Calcul du spectre d'une nilvariete de rang deux et applications, Trans. Amer. Math. Soc. 339 (1993), 433-461.

[Rh] C. RiEHm, Explicit spin representation and Lie algebras of Heisenberg type, J. London Math. Soc. 29 (1984), 49-62.

[Sch] D. Schueth, Continuous families of isospectral metrics on simply connected manifolds, Ann.of Math. 149 (1999), 287-308.

[S] T. SunadA, Riemannian coverings and isospectral manifolds, Ann. of Math. (2)121 (1985), 169-186.

[Sz1] Z. I. Szabó, Lichnerowicz conjecture on harmonic manifolds, J. Diff. Geom. 31 (1990), 1-28.

[Sz2] Z. I. Szabó, Spectral theory for operator families on Riemannian Manifolds, Proc. of Symp. in Pure Math. vol. 54 (1993), Part 3, 615-665.

[Sz3] Z. I. Szabó, Locally non-isometric yet super isospectral spaces, Geom. funct. anal. (GAFA) 9 (1999 (in preprint 1992)), 185-214.

[Sz4] Z. I. Szabó, Cornucopia of isospectral pairs of metrics on balls and spheres with different local geometries, Preprint (2000).

[V] M.-F. Vigneras, Varietes Riemanniennes isospectrales et non isometrique, Ann. of Math. (2)112 (1980), 21-32.

[W] E. N. Wilson, Isometry groups on homogeneous nilmanifolds, Geom. Dedicata 12 (1982), 337-346.

Zoltán IMRe Szabó

CUny, Lehman College, Bronx, Ny 10468-1589, USA

Rényi Alfréd Institute of Math., P. O. Box 127, H-1053 Budapest, Hungary

EMAIL: zoltan@alpha.lehman.cuny.edu 